\title{
Topology optimization of frequency dependent viscoelastic structures via a level-set method
}

\author{
G. Delgado \\ IRT SystemX, Paris-Saclay, France \\ M. Hamdaoui \\ LeM3, Université de Lorraine, 7 Rue Félix Savart, 57000 Metz, France
}

\begin{abstract}
Viscoelastic materials follow a liquid-like elastic behavior whose characteristics depend on the excitation frequency. Nowadays, this type of materials represent a high opportunity for vibration damping treatments in the automotive and aeronautic industries, for instance. This work is devoted to the application of the level-set method for topology optimization of viscoelastic structures. We look for the best distribution of viscoelastic material within a reference domain for the design of purely viscoelastic 3D damping structures and $2 \mathrm{D}$ viscoelastic damping treatments. In both cases one desires to maximize the structure capacity to dissipate energy measured here by the modal loss factor of the first vibration mode.
\end{abstract}

Keywords: Topology optimization, Level-set method, Viscoelastic damping, Non-linear eigenvalue problem

\section{Introduction}

Viscoelastic damping material behavior occurs in a wide variety of materials and can be characterized by liquid-like elastic behavior. Materials that experience viscoelastic behavior include acrylics, rubber, and adhesives. The characteristics of viscoelastic materials depend on temperature and frequency. Viscoelastic materials have the property of absorbing vibrational energy which makes them very interesting for structural damping applications. The vibrations can be caused by noises that radiate from a certain source as sound or structure oscillations coming from dynamical wind loadings or earthquakes. Viscoelastic dampers can be designed as independent 3D parts within a mechanism but also as 2D damping treatments which consist of one or a combination of materials applied/bonded to a component to increase its ability to dissipate mechanical energy. For instance, viscoelastic dampers are useful when structures are forced to vibrate at or near their natural (resonant) frequencies, are subjected to impacts or other transient forces, or transmit vibrations to noise-radiating surfaces. The energy dissipation of a viscoelastic structure is typically quantified in terms of a loss factor, a dimensionless quantity that can be measured or predicted from a modal analysis or frequency response curves. Two categories of treatments for structural damping exist, the unconstrained layer damping (UCLD), where the material is simply attached with a strong bonding agent to the surface of a structure, and Constrained-layer damping (CLD) where the material is sandwiched between a base layer and a third constraining layer. The base layer refers to the structure to be coated. The CLD treatment provides considerably more damping effect than the free viscoelastic treatment in spite of its relative complexity. Energy dissipation is achieved in this case by shearing a viscoelastic polymer between a base structure and a constraining layer. Both types of damping structures are depicted in Figures 1 and 2.

Topology optimization is essentially an iterative numerical process that seeks to find the best material layout (within a prescribed design domain) according to a given objective function and a set of design constraints, providing valuable help in problems where mechanical intuition is limited. Topology optimization of viscoelastic UCLD and CLD has been performed by many authors in the literature using different methods. Zheng et al. [1] used the Solid Isotropic Material with Penalization (SIMP) method with the 


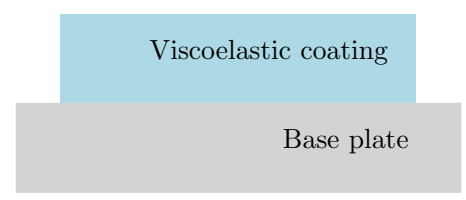

Figure 1: Unconstrained-layer damping treatment.

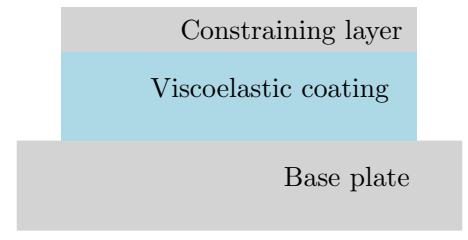

Figure 2: Constrained-layer damping treatment.

Method of Moving Asymptote (MMA) to perform topology optimization of a CLD cantilever plate treated with DYAD606 where a sum of modal loss factors is maximized. Kim 2 applied SIMP and Evolutionary Structural Optimization (ESO) methods to design damping treatments for unconstrained-layer plate and shell structures. Zheng et al. 3] used the same methodology to perform topology optimization of CLD with partial coverage, showing interesting performances of the optimized structure in terms of damping and mass savings. Kim et al. [4 used the rational approximation for material properties (RAMP) with the optimality criteria method (OC) to perform topology optimization of UCLD shell structures to maximize modal loss factors. El-Sabbagh et al. [5] used the method presented in [6] along with the MMA method to perform optimization of periodic and non-periodic plates. Zhanpeng et al. 7] used evolutionary structural optimization (ESO) to minimize viscoelastic CLD plate response. James et al. 8 used a time dependent adjoint method along with the MMA method to perform topology optimization of viscoelastically damped beams for minimum mass under time dependent loadings. Yun et al. 9] performed multimaterial topology optimization to maximize energy dissipation of viscoelastically damped structures subjected to unsteady loads using SIMP and MMA. Ansari et al. 10, used a level set method to perform topology optimization of viscoelastic UCLD plate whereas van der Kolk et al. 11 used a parametrized level set based method for multi-material topology optimization of beams.

The present work addresses the structural optimization of 3D fully viscoelastic structures and 2D UCLD treatments by means of the level-set method for topology optimization. For this purpose we rely on the level-set approach for multi-phase optimization detailed by Allaire et al. [12. First introduced by Osher and Sethian [13, the level-set method has the advantage of tracking the interfaces on a fixed mesh, easily managing topological changes without remeshing. Combined to the Hadamard method of shape differentiation [14, 15, 16] in the framework of structural optimization, the level-set approach is an efficient shape and topology optimization algorithm, which gives a better description and control of the geometrical properties of the interface, avoiding typical drawbacks such as intermediate density penalization and possible spurious physical behavior during the optimization process [17, 18. Moreover, as remarked by Allaire et al. [19], the level-set method is especially well suited for vibration problems involving eigenfrequency optimization, since small holes or material islands cannot suddenly appear or disappear between two successive iterations as they do with the homogenization or SIMP methods, thus avoiding spurious modes in low density regions. Indeed, the "ersatz material approach" used within the framework of the above mentioned methods is known to produce fictitious eigenmodes localized in the weak phase which pollute the optimization process (see e.g. [20], 6], 21]).

This article is organized as follows. Section 2 is devoted to the mathematical description of viscoelastic materials and the vibration problem arising for purely $3 \mathrm{D}$ viscoelastic structures and $2 \mathrm{D}$ unconstrained layer damping plates. Both cases are associated to two different models involving viscoelasticity and are dealt with in parallel inasmuch as possible. Section 3 is concerned with the study of the non-linear eigenvalue problem that stems from the aforementioned models. We provide a theoretical result characterizing the 
eigenvalues of such problems for a general class of viscoelastic materials. Section 4 details the sensitivity analysis of the first eigenfrequency of the structure with respect to the viscoelastic shape, and Section 5 recalls the use of the level-set method in the framework of topology optimization. Finally Section 6 outlines the numerical solution of the underlying non-linear eigenvalue problem and illustrates it with two concrete applications.

\section{Setting of the problem}

Let $\hat{f}$ be the Laplace transform of a real-valued function $f(t)$ defined by

$$
\hat{f}(\omega)=\int_{0}^{\infty} e^{-i \omega t} f(t) d t, \omega \in \mathbb{C} .
$$

From now on, we will assume a time-harmonic regime at fixed $\omega$. For viscoelastic materials, a linear elastic constitutive relationship using Hooke's law is not an accurate model and a complex modulus is extensively used to describe the dynamic characteristics of viscoelastic materials [22]. The stress-strain relationship of a linear viscoelastic damping material subjected to steady-state oscillatory loads thus reads:

$$
\hat{\sigma}(\omega)=\hat{A}(\omega) \hat{\epsilon}(\omega)
$$

where $\omega$ is the complex pulsation, $\hat{A}$ is the complex (or dynamic) elastic tensor and $\hat{\sigma}$ and $\hat{\epsilon}$ are the Laplace transforms of stress and the strain, respectively. This relation stems from the Laplace transform of the stress history given by

$$
\sigma(t)=\int_{0}^{t} Y(t-\tau) \frac{d \epsilon(\tau)}{d \tau} d \tau,
$$

with $Y$ the relaxation function (or relaxation modulus) and

$$
\hat{A}=i \omega \hat{Y}(\omega)
$$

The relaxation function $Y$ accounts for the material stress behavior in a relaxation test (gradual disappearance of stresses from a viscoelastic medium after it has been deformed) and the Boltzmann superposition principle (the state of stress or deformation of a viscoelastic body is a function of all the stresses and/or deformations applied to the material) [23. In other words, the relaxation function $Y$ governs the decrease of the stress towards an asymptotical limit in time when the viscoelastic material is subjected to an instantaneous constant strain at $t=0$. Then for a sequence $d \epsilon(t)$ of applied strains in time, the stress is simply equal to the "sum of products" of the delayed relaxation function and the constant applied strains. This integral is later expressed rather as a convolution.

For the sake of simplicity, we will henceforth write $(A, \sigma, \epsilon)$ instead of $(\hat{A}, \hat{\sigma}, \hat{\epsilon})$ unless we want to explicitly emphasize the Laplace transform.

Let $(\Omega, \mathcal{O})$ be two bounded open sets with $\Omega \subset \mathcal{O} \subset \mathbb{R}^{d}(d \in\{2,3\})$.

- If $d=3$, we will say that $\Omega$ is occupied by a viscoelastic material with complex elastic tensor $A$ and density $\rho>0$ within a reference (or working) domain $\mathcal{O}$. We will call this configuration the $3 D$ viscoelastic structure. The boundary of $\Omega$ is made of two disjoint parts

$$
\partial \Omega=\Gamma_{N} \cup \Gamma_{D}
$$

with Dirichlet boundary conditions on $\Gamma_{D}$ and Neumann boundary conditions on $\Gamma_{N}$.

- If $d=2, \mathcal{O}$ will be the reference configuration for a plate in the $x y$-plane and $\Omega$ the surface occupied by the viscoelastic coating. We will call this configuration the composite sandwich structure. By abuse of notation, we split the boundary of $\mathcal{O}\left(\partial \mathcal{O}=\Gamma_{N} \cup \Gamma_{D}\right)$ instead of $\Omega$ since no boundary conditions are imposed on $\partial \Omega$. 


\subsection{D viscoelastic structure}

We denote by $\omega \in \mathbb{C}$ the complex pulsation and by $\hat{u} \in H^{1}(\Omega ; \mathbb{C})^{3}$ the associated mode, i.e. the corresponding displacement field in $\Omega$. For the sake of simplicity, we will generally write $u$ unless we want to explicitly emphasize the Laplace transform. The pair $(\omega, u)$ is a solution of the non-linear eigenvalue problem

$$
\left\{\begin{array}{cl}
-\operatorname{div}(A(\omega) e(u))=\omega^{2} \rho u & \text { in } \Omega, \\
u=0 & \text { on } \Gamma_{D}, \\
A(\omega) e(u) \cdot n=0 & \text { on } \Gamma_{N},
\end{array}\right.
$$

where

$$
e(u)=\frac{\nabla u+\nabla u^{T}}{2}
$$

is the symmetrized strain tensor and $n$ is the normal vector. The state equation in (5) stems from applying the constitutive equation (2) and the Laplace transform to the evolution (wave) equation of linear elasticity

$$
\rho u_{t t}-\operatorname{div}(\sigma)=0 \text { in } \Omega .
$$

We remark that the eigenvalue problem (5) is non-linear since the complex elastic (or stiffness) tensor $A$ also depends on $\omega$.

\subsection{Composite sandwich structure}

For modeling vibration damping treatments we use a plate model approximation of (5). Consider the small transverse vibration of a uniform plate with thickness $h(x)$, density $\rho(x)$ and reference configuration for the plate $\mathcal{O}$ with $d=2$. The transverse displacement of $x$ at time $t$ is denoted as $\mathrm{w}(x, t)$ but for the sake of simplicity, we rather denote as w (instead of $\hat{w}$ ) the vertical displacement mode associated with the complex pulsation $\omega \in \mathbb{C}$. Unconstrained-layer damping (UCLD) is one of the simplest forms of material application. The material is simply attached with a strong bonding agent to the surface of a structure. Energy is dissipated as a result of extension and compression of the damping material under flexural stress from the base structure. From now on, for every general quantity $\xi$ defined either on the viscoelastic coating, the base plate or the global composite (viscoelastic coating plus base plate), we will use the notations $\xi_{c}, \xi_{p}, \xi_{g}$ respectively. The following assumptions are made:

- Transverse shear and rotational and in-plane inertia effects in both the plate and the coating are negligible for the lower modes of vibration,

- There are no applied in-plane loads,

- Displacements are small and changes in thickness are negligible,

- The viscoelastic coating is applied to only one side of the plate

- The plate and the coating are homogeneous and isotropic, and subjected to a state of plane stress,

- Displacements are continuous across the interface between the plate and the coating,

- The Poisson's ratio of the coating is a real constant and the coating is incompressible $(\nu=0.5)$.

Under these assumptions, classical Kirchhoff-Love plate theory can be used [24]. It is worth mentioning that the plate is considered to be in a free vibration state. Let the pair $(\omega, \mathrm{w})$, with w $\in H^{2}(\mathcal{O} ; \mathbb{C})$, be the solution of the non-linear eigenvalue problem

$$
\left\{\begin{array}{cl}
-\nabla^{2}:\left(D(\omega) \nabla^{2} \mathrm{w}\right)=\omega^{2} \rho \mathrm{w} & \text { in } \mathcal{O}, \\
\mathrm{w}=0, \nabla \mathrm{w}=0 & \text { on } \Gamma_{D}, \\
n \cdot\left(D(\omega) \nabla^{2} \mathrm{w}\right) \cdot n=0 & \text { on } \Gamma_{N} .
\end{array}\right.
$$




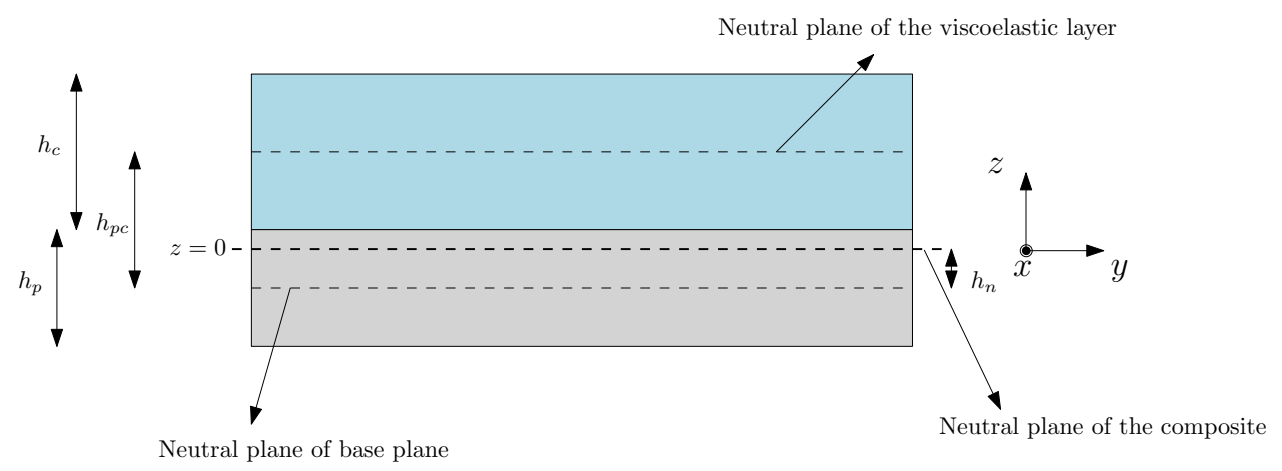

Figure 3: Slice of the $3 \mathrm{~d}$ plate. The shape $\mathcal{O}$ belongs to the $x y$-plane, being $x$ the outward axis in the figure.

where $\nabla^{2}$ is the second order partial derivatives operator. The aforementioned state equation follows from the virtual work principle applied to the total energy of the plate (bending strain energy plus kinetic energy) and the Laplace transform. As mentioned before, let $\Omega$ be the region of $\mathcal{O}$ where the coating is applied. Then the function $\rho(x)$ is piecewise constant and corresponds to the surface density

$$
\rho(x)= \begin{cases}\rho_{p}:=\rho_{p}^{V} h_{p} & \text { if } x \in \mathcal{O} \backslash \Omega, \\ \rho_{g}:=\left(\rho_{p}^{V} h_{p}+\rho_{c}^{V} h_{c}\right) & \text { if } x \in \Omega\end{cases}
$$

with $\rho_{p}^{V}, \rho_{c}^{V}$ and $h_{p}, h_{c}$ the volumetric densities and thicknesses of the plate and the viscoelastic coating, respectively. The equivalent bending stiffness tensor $D$ for an isotropic base and coating material reads [25]

$$
D(\omega, x)= \begin{cases}D_{p}:=\frac{h_{p}^{3}}{12} A_{p} & \text { if } x \in \mathcal{O} \backslash \Omega, \\ D_{g}:=\left(\frac{h_{p}^{3}}{12}+h_{p} h_{n}^{2}\right) A_{p}+\left(\frac{h_{c}^{3}}{12}+h_{c}\left(h_{p c}-h_{n}\right)^{2}\right) A_{c}(\omega) & \text { if } x \in \Omega,\end{cases}
$$

where the tensors $A_{p}, A_{c}(\omega)$ stand for the extensional stiffness tensors of the base and viscoelastic materials respectively (we remark that only the coating material depends on $\omega$, more specifically its Young modulus),

$$
A_{l}=\frac{E_{l}}{\left(1-\nu_{l}^{2}\right)}\left(\begin{array}{ccc}
1 & \nu_{l} & 0 \\
\nu_{l} & 1 & 0 \\
0 & 0 & 0.5\left(1-\nu_{l}\right)
\end{array}\right), \quad \text { with the index } l=p, c,
$$

$h_{p c}$ represents the distance between the neutral planes of the base plate and the viscoelastic layer material and $h_{n}$ (see Figure 3 represents the distance between the composite neutral axis and the base plate neutral plane which can be approximated as

$$
h_{n}=\frac{E_{c} h_{c} h_{p c}}{E_{p} h_{p}+E_{c} h_{c}}
$$

The structure of the tensor $D$ ensues from the integration of the laminate through the thickness moment resultants meanwhile the correction terms $h_{n}, h_{p c}$ arise when $h_{p} \neq h_{c}$ in order to compensate for the shift of the neutral fiber of the laminate, i.e. the place in the plate where the thickness-averaged stresses vanish 25].

\subsection{Optimization problem}

For the sake of clarity, we detail the optimization problem only within the damping optimization context exposed in Section 2.1. The same notions however remain valid for viscoelastic damping treatments. Supposing that (5) admits a countably infinite family of solutions $\left(\omega_{k}, u_{k}\right)_{k \geq 1}$ in $\mathbb{C} \times H^{1}(\Omega \text {; } \mathbb{C})^{3}$ (see more 
details in the next section), with the eigenfunctions or modes normalized by imposing that $\int_{\Omega} \rho\left|u_{k}\right|^{2} d x=1$, we are interested in solving the optimization problem

$$
\sup _{\Omega \in \mathcal{U}_{a d}} \eta(\Omega)
$$

where

$$
\mathcal{U}_{a d}=\{\Omega \subset \mathcal{O} \text { such that }|\Omega|=\mathcal{V}\}
$$

is the set of admissible shapes with fixed volume $\mathcal{V}, \mathcal{O}$ is the working domain (a bounded subset of $\mathbb{R}^{3}$ ) and $\eta(\Omega)$ is the modal loss factor of the structure for its first eigenvalue:

$$
\eta(\Omega):=\frac{\operatorname{Im}\left(\omega_{1}^{2}\right)}{\operatorname{Re}\left(\omega_{1}^{2}\right)}=\frac{\operatorname{Im}\left(\int_{\Omega} A e\left(u_{1}\right): e\left(\bar{u}_{1}\right) d x\right)}{\operatorname{Re}\left(\int_{\Omega} A e\left(u_{1}\right): e\left(\bar{u}_{1}\right) d x\right)},
$$

where $\bar{u}$ is the complex conjugate of $u$. The value of $\eta$ represents the ratio between dissipated and stored energy or in other words, the structure capacity to dissipate energy. It is obviously a measure of the structure mechanical damping [26].

Remark 2.1. It is well-known that (11) is usually not well posed on the set of admissible shapes (12) (i.e. it has no solution). In order to obtain existence of optimal shapes, some smoothness or geometrical or topological constraints are required (see for instance [27, 28, 29] ). In the present article, we shall work with a subset of 12 composed of smoother shapes in order to define properly the notion of shape derivative (consult Section 4).

Similarly to the notion of material loss factor of a viscoelastic material, which allows to express the dissipative behavior of the complex Young and shear moduli $E(\omega), G(\omega)$ via the Young and shear moduli factors $\eta_{E}, \eta_{G}$ :

$$
E=\operatorname{Re}(E)\left(1+i \eta_{E}\right) \quad \text { and } \quad G=\operatorname{Re}(G)\left(1+i \eta_{G}\right),
$$

the concept of modal loss factor accounts for the dissipative behavior of the structure first resonant frequency

$$
\omega_{1}^{2}=\operatorname{Re}\left(\omega_{1}^{2}\right)(1+i \eta) .
$$

Since the eigenvalues of (5) cannot be naturally ordered in $\mathbb{C}, \omega_{1}$ will be defined as the closest eigenvalue of (5) to $\omega_{1}^{\ell}$ (in the sens of modulus $\left.\left|\omega_{1}-\omega_{1}^{\ell}\right|\right)$, where $\left(\omega_{k}^{\ell}, u_{k}^{\ell}\right)_{k \geq 1}$ in $\mathbb{R} \times H^{1}(\Omega ; \mathbb{R})^{3}$ and $\omega_{k}^{\ell} \leq \omega_{k+1}^{\ell}$ for all $k \geq 1$, is the countably infinite family of solutions of the purely elastic problem associated with (5):

$$
-\operatorname{div}(A(0) e(u))=\omega^{2} \rho u \text { in } \Omega .
$$

\section{The non-linear eigenvalue problem}

Problem (5) can be cast as a generalized eigenvalue problem

$$
\mathcal{T}(\omega) u=0
$$

where $\mathcal{T}(\omega)$ is a linear operator depending (non-linearly) on a parameter $\omega$. In particular for (5)

$$
\mathcal{T}(\omega)=-\operatorname{div}(A(\omega) e(\cdot))-\omega^{2} \rho \operatorname{Id}(\cdot),
$$

where $\operatorname{Id}()$ is the identity operator. A solution $u \neq 0$ will exist only for some particular values of $\omega$ (also called eigenvalues).

We devote this section to the study of the characterization of the solutions of (5) and (7). We prove in particular that the underlying spectrum of the aforementioned problems is discrete. This feature is of paramount importance since a continuous spectrum or an accumulation point in the spectrum may lead to spurious eigenvalues in numerical applications. 


\subsection{Preliminary results}

Let $\mathbb{V}$ and $\mathbb{W}$ be two complex Hilbert spaces with $\mathbb{V}$ compactly embedded in $\mathbb{W}$. Let also $\left\{\mathcal{E}_{\omega}\right\}_{\omega \in \mathbb{D}}$, with $\mathbb{D} \subset \mathbb{C}$ open bounded, be a family of complex mappings $\mathcal{E}_{\omega}: \mathbb{V} \times \mathbb{V} \rightarrow \mathbb{C}$.

Definition 3.1 (Sesquilinear form). We say that $\left\{\mathcal{E}_{\omega}\right\}_{\omega \in \mathbb{D}}$ is a family of sesquilinear forms if for each $\omega$

1. $\mathcal{E}_{\omega}\left(u_{1}+u_{2}, v_{1}+v_{2}\right)=\mathcal{E}_{\omega}\left(u_{1}, v_{1}\right)+\mathcal{E}_{\omega}\left(u_{1}, v_{2}\right)+\mathcal{E}_{\omega}\left(u_{2}, v_{1}\right)+\mathcal{E}_{\omega}\left(u_{2}, v_{2}\right)$,

2. $\mathcal{E}_{\omega}\left(\alpha v_{1}, \beta v_{2}\right)=\alpha \bar{\beta} \mathcal{E}_{\omega}\left(v_{1}, v_{2}\right)$,

for all $u_{1}, u_{2}, v_{1}, v_{2} \in \mathbb{V}$ and all $\alpha, \beta \in \mathbb{C}$.

Definition 3.2 (Continuous form). We say that $\left\{\mathcal{E}_{\omega}\right\}_{\omega \in \mathbb{D}}$ is a family of continuous forms if there exists a function $d: \mathbb{D} \rightarrow \mathbb{R}^{+}$such that

$$
\left|\mathcal{E}_{\omega}\left(v_{1}, v_{2}\right)\right| \leq d(\omega)\left\|v_{1}\right\|_{\mathbb{V}}\left\|v_{2}\right\|_{\mathbb{V}}, \forall v_{1}, v_{2} \in \mathbb{V}
$$

We will suppose additionally that the function $d(\omega)$ is continuous in $\overline{\mathbb{D}}$ (thus bounded).

Definition 3.3 (Coercive form). We say that $\left\{\mathcal{E}_{\omega}\right\}_{\omega \in \mathbb{D}}$ is a family of coercive forms if there exists a function $c: \mathbb{D} \rightarrow \mathbb{R}^{+}$such that

$$
\left|\mathcal{E}_{\omega}(v, v)\right| \geq c(\omega)\|v\|_{\mathbb{V}}^{2}, \forall v \in \mathbb{V} .
$$

We will suppose additionally that the function $c(\omega)$ is continuous and $c(\omega) \geq \alpha>0$ in $\overline{\mathbb{D}}$ (thus bounded away from 0).

Definition 3.4 (Holomorphic form). We say that $\left\{\mathcal{E}_{\omega}\right\}_{\omega \in \mathbb{D}}$ is a family of holomorphic forms if for every fixed pair $v_{1}, v_{2} \in \mathbb{V}$ the mapping

$$
\omega \in \mathbb{C} \rightarrow \mathcal{E}_{\omega}\left(v_{1}, v_{2}\right) \in \mathbb{C}
$$

is differentiable at $\omega$ for every point $\omega$ in $\mathbb{D}$.

From now on, we will assume that $\left\{\mathcal{E}_{\omega}\right\}_{\omega \in \mathbb{D}}$ is a family of continuous coercive sesquilinear forms with continuous bounds.

Proposition 3.1. For each $\omega \in \mathbb{D}$, define the operators $\mathcal{G}_{\omega}, \mathcal{G}_{\omega}^{\dagger}: \mathbb{W} \rightarrow \mathbb{W}$ such that for given $g \in \mathbb{W}, \mathcal{G}_{\omega}(g)$ and $\mathcal{G}_{\omega}^{\dagger}(g)$ are respectively the solutions of

$$
\begin{aligned}
& \mathcal{E}_{\omega}\left(\mathcal{G}_{\omega}(g), v\right)=\langle g, v\rangle_{\mathbb{W}}, \forall v \in \mathbb{V} \\
& \mathcal{E}_{\omega}\left(v, \mathcal{G}_{\omega}^{\dagger}(g)\right)=\langle g, v\rangle_{\mathbb{W}}, \forall v \in \mathbb{V} .
\end{aligned}
$$

Then for each $\omega \in \mathbb{D}$, the operators $\mathcal{G}_{\omega}, \mathcal{G}_{\omega}^{\dagger}$ are well-defined and compact.

Proof. We only give the proof for $\mathcal{G}_{\omega}$ as the argument is analogous for $\mathcal{G}_{\omega}^{\dagger}$. According to (17) and (18), we can apply the Lax-Milgram theorem to 1 aa) so for a given $g \in \mathbb{W}$, there exists a unique solution $\mathcal{G}_{\omega}(g) \in \mathbb{V} \subset \mathbb{W}$. Furthermore, since $\mathbb{V}$ is compactly embedded in $\mathbb{W}$, there exists a constant $b>0$ such that

$$
b\left\|\mathcal{G}_{\omega}(g)\right\|_{\mathbb{W}} \leq\left\|\mathcal{G}_{\omega}(g)\right\|_{\mathbb{V}},
$$

so the operator $\mathcal{G}_{\omega}$ is continuous with

$$
\left\|\mathcal{G}_{\omega}(g)\right\|_{\mathbb{W}} \leq \frac{1}{b^{2} \alpha}\|g\|_{\mathbb{W}}
$$

thanks to Cauchy-Schwarz inequality and the Lax-Milgram estimate of 19a). 
Proposition 3.2. Assume that $\left\{\mathcal{E}_{\omega}\right\}_{\omega \in \mathbb{D}}$ is a family of holomorphic forms and for $\omega$ fixed, the first and second derivative mappings of $\mathcal{E}_{\omega}$, denoted respectively as

$$
\partial_{\omega} \mathcal{E}_{\omega}: \mathbb{V} \times \mathbb{V} \rightarrow \mathbb{C}, \quad \partial_{\omega}^{2} \mathcal{E}_{\omega}: \mathbb{V} \times \mathbb{V} \rightarrow \mathbb{C},
$$

are continuous sesquilinear forms with continuous bounds. For every $\omega \in \mathbb{D}$, define the complex mapping $\mathcal{F}_{\omega}: \mathbb{W} \times \mathbb{W} \rightarrow \mathbb{C}$ as

$$
\mathcal{F}_{\omega}\left(v_{1}, v_{2}\right)=\left\langle\mathcal{G}_{\omega}\left(v_{1}\right), v_{2}\right\rangle_{\mathbb{W}}, v_{1}, v_{2} \in \mathbb{W},
$$

where the operator $\mathcal{G}_{\omega}$ is defined in 19a. Then $\left\{\mathcal{F}_{\omega}\right\}_{\omega \in \mathbb{D}}$ is a family of holomorphic forms.

Proof. We will prove that for an arbitrary fixed pair $v_{1}, v_{2} \in \mathbb{W}$, the mapping

$$
\omega \in \mathbb{D} \rightarrow \mathcal{F}_{\omega}\left(v_{1}, v_{2}\right)=\left\langle\mathcal{G}_{\omega}\left(v_{1}\right), v_{2}\right\rangle_{\mathbb{W}} \in \mathbb{C}
$$

is differentiable at $\omega_{0}$ for every $\omega_{0} \in \mathbb{D}$. Let $p_{\omega_{0}} \in \mathbb{V}$ be the solution of

$$
\mathcal{E}_{\omega_{0}}\left(p_{\omega_{0}}, v\right)=-\partial_{\omega} \mathcal{E}_{\omega_{0}}\left(\mathcal{G}_{\omega_{0}}\left(v_{1}\right), v\right), \forall v \in \mathbb{V}
$$

for a given $v_{1} \in \mathbb{V}$. The existence and uniqueness of $p_{\omega_{0}}$ stem from the Lax-Milgram theorem according to (17), 18 and the continuity of the operator $\partial_{\omega} \mathcal{E}_{\omega_{0}}$. Let $\Delta \omega \in \mathbb{C}$ be small enough so $\omega_{0}+\Delta \omega \in \mathbb{D}$. Then 22 is differentiable at $\omega_{0}$ with

$$
\lim _{\Delta \omega \rightarrow 0}\left|\frac{\left\langle\mathcal{G}_{\omega_{0}+\Delta \omega}\left(v_{1}\right), v_{2}\right\rangle_{\mathbb{W}}-\left\langle\mathcal{G}_{\omega_{0}}\left(v_{1}\right), v_{2}\right\rangle_{\mathbb{W}}}{\Delta \omega}-\left\langle p_{\omega_{0}}\left(v_{1}\right), v_{2}\right\rangle_{\mathbb{W}}\right|=0 .
$$

Indeed

$$
\begin{aligned}
& \left|\frac{\left\langle\mathcal{G}_{\omega_{0}+\Delta \omega}\left(v_{1}\right), v_{2}\right\rangle_{\mathbb{W}}-\left\langle\mathcal{G}_{\omega_{0}}\left(v_{1}\right), v_{2}\right\rangle_{\mathbb{W}}}{\Delta \omega}-\left\langle p_{\omega_{0}}\left(v_{1}\right), v_{2}\right\rangle_{\mathbb{W}}\right| \\
= & \frac{1}{|\Delta \omega|} \mid\left\langle\mathcal{G}_{\omega_{0}+\Delta \omega}\left(v_{1}\right)-\mathcal{G}_{\omega_{0}}\left(v_{1}\right)-\Delta \omega p_{\omega_{0}}\left(v_{1}\right), v_{2}\right\rangle \\
= & \frac{1}{|\Delta \omega|}\left|\left\langle v_{2}, \mathcal{G}_{\omega_{0}+\Delta \omega}\left(v_{1}\right)-\mathcal{G}_{\omega_{0}}\left(v_{1}\right)-\Delta \omega p_{\omega_{0}}\left(v_{1}\right)\right\rangle_{\mathbb{W}}\right| \\
= & \frac{1}{|\Delta \omega|}\left|\mathcal{E}_{\omega_{0}+\Delta \omega}(\underbrace{\mathcal{G}_{\omega_{0}+\Delta \omega}\left(v_{1}\right)}_{\text {first member }} \underbrace{-\mathcal{G}_{\omega_{0}}\left(v_{1}\right)-\Delta \omega p_{\omega_{0}}\left(v_{1}\right)}_{\text {second member }}, \mathcal{G}_{\omega_{0}+\Delta \omega}^{\dagger}\left(v_{2}\right))\right| .
\end{aligned}
$$

Then developing each member of 25] :

$$
\mathcal{E}_{\omega_{0}+\Delta \omega}\left(\mathcal{G}_{\omega_{0}+\Delta \omega}\left(v_{1}\right), \mathcal{G}_{\omega_{0}+\Delta \omega}^{\dagger}\left(v_{2}\right)\right)=\left\langle v_{1}, \mathcal{G}_{\omega_{0}+\Delta \omega}^{\dagger}\left(v_{2}\right)\right\rangle_{\mathbb{W}}
$$

according to $19 \mathrm{a}$ and

$$
\begin{aligned}
-\mathcal{E}_{\omega_{0}+\Delta \omega}\left(\mathcal{G}_{\omega_{0}}\left(v_{1}\right)+\Delta \omega p_{\omega_{0}}\left(v_{1}\right), \mathcal{G}_{\omega_{0}+\Delta \omega}^{\dagger}\left(v_{2}\right)\right) & =-\mathcal{E}_{\omega_{0}}\left(\mathcal{G}_{\omega_{0}}\left(v_{1}\right)+\Delta \omega p_{\omega_{0}}\left(v_{1}\right), \mathcal{G}_{\omega_{0}+\Delta \omega}^{\dagger}\left(v_{2}\right)\right) \\
& -\Delta \omega \partial_{\omega} \mathcal{E}_{\omega_{0}}\left(\mathcal{G}_{\omega_{0}}\left(v_{1}\right)+\Delta \omega p_{\omega_{0}}\left(v_{1}\right), \mathcal{G}_{\omega_{0}+\Delta \omega}^{\dagger}\left(v_{2}\right)\right) \\
& -(\Delta \omega)^{2} \partial_{\omega}^{2} \mathcal{E}_{\omega_{0}}\left(\mathcal{G}_{\omega_{0}}\left(v_{1}\right)+\Delta \omega p_{\omega_{0}}\left(v_{1}\right), \mathcal{G}_{\omega_{0}+\Delta \omega}^{\dagger}\left(v_{2}\right)\right)(29) \\
& +O\left((\Delta \omega)^{3}\left\|v_{1}\right\|_{\mathbb{V}}\left\|v_{2}\right\|_{\mathbb{V}}\right)
\end{aligned}
$$


according to the second-order Taylor expansion of $\mathcal{E}_{\omega}$ with respect to $\omega$ (by hypothesis we assume the mapping $\omega \rightarrow \mathcal{E}_{\omega}\left(v_{1}, v_{2}\right)$ holomorphic for $\left.\omega \in \mathbb{D}\right)$. By virtue of (19a) and (23), 27) satisfies

$$
-\mathcal{E}_{\omega_{0}}\left(\mathcal{G}_{\omega_{0}}\left(v_{1}\right)+\Delta \omega p_{\omega_{0}}\left(v_{1}\right), \mathcal{G}_{\omega_{0}+\Delta \omega}^{\dagger}\left(v_{2}\right)\right)=-\left\langle v_{1}, \mathcal{G}_{\omega_{0}+\Delta \omega}^{\dagger}\left(v_{2}\right)\right\rangle_{\mathbb{W}}+\Delta \omega \partial_{\omega} \mathcal{E}_{\omega_{0}}\left(\mathcal{G}_{\omega_{0}}\left(v_{1}\right), \mathcal{G}_{\omega_{0}+\Delta \omega}^{\dagger}\left(v_{2}\right)\right),
$$

so adding (26), 27), 28) and (29), a few terms cancel and we deduce that 25 is equal to

$$
\begin{aligned}
& =\frac{1}{|\Delta \omega|}\left|-(\Delta \omega)^{2}\left\{\partial_{\omega} \mathcal{E}_{\omega_{0}}\left(p_{\omega_{0}}\left(v_{1}\right), \mathcal{G}_{\omega_{0}+\Delta \omega}^{\dagger}\left(v_{2}\right)\right)+\partial_{\omega}^{2} \mathcal{E}_{\omega_{0}}\left(\mathcal{G}_{\omega_{0}}\left(v_{1}\right), \mathcal{G}_{\omega_{0}+\Delta \omega}^{\dagger}\left(v_{2}\right)\right)\right\}+O\left((\Delta \omega)^{3}\left\|v_{1}\right\|_{\mathbb{V}}\left\|v_{2}\right\|_{\mathbb{V}}\right)\right| \\
& \leq O\left(|\Delta \omega|\left\|v_{1}\right\|_{\mathbb{V}}\left\|v_{2}\right\|_{\mathbb{V}}\right),
\end{aligned}
$$

where the last inequality stems from boundedness in $\overline{\mathbb{D}}$ of the continuity bounds of $\partial_{\omega} \mathcal{E}_{\omega}, \partial_{\omega}^{2} \mathcal{E}_{\omega}$ and the Lax-Milgram bounds derived in the proof of Proposition 3.1 (reasoning by analogy, the same bounds can be applied to (23)). Finally taking the limit when $\Delta \omega \rightarrow 0$ yields (24).

\subsection{Main result}

The main conclusion of this section corresponds to the application of a result of standard analytical perturbation theory of compact operators. This theorem describes locally the eigenvalues of a general non-linear eigenvalue problem

Theorem 3.1 (30, chapter VII, Th. 1.9). Let $\mathcal{S}(\omega)$ be a family of compact operators (for each $\omega$ fixed) and holomorphic with respect to $\omega \in \mathbb{D} \subset \mathbb{C}$ bounded. Define $\omega$ as a singular point if 1 is an eigenvalue of $\mathcal{S}(\omega)$. Then either all $\omega \in \mathbb{D}$ are singular points or there are only a finite number of singular points in each compact subset of $\mathbb{D}$.

Theorem 3.2. Suppose that the weak formulation of (16) has the following structure on $\omega$

$$
\mathcal{E}_{\omega}(u, v)-\omega^{2}\langle u, v\rangle_{\mathbb{W}}=0, \forall v \in \mathbb{V}
$$

and assume the same hypotheses as Proposition 3.1 and Proposition 3.2. Furthermore suppose that $0 \in \mathbb{D}$ and $\mathcal{E}_{0}$ hermitian, i.e.

$$
\mathcal{E}_{0}(u, v)=\overline{\mathcal{E}_{0}(v, u)}, \forall u, v \in \mathbb{V} .
$$

Then there are only a finite number of eigenvalues of $(30)$ in $\mathbb{D}$ (i.e. values of $\omega$ for which there exist $u \neq 0$ solution of (30)).

Proof. Define the operator $\mathcal{S}_{\omega}: \mathbb{W} \rightarrow \mathbb{W}$ as

$$
\mathcal{S}_{\omega}=\omega^{2} \mathcal{G}_{\omega}
$$

Then according to Proposition 3.1 and Proposition $3.2\left\{S_{\omega}\right\}_{\omega \in \mathbb{C}}$ is a family of compact operators and $\mathcal{S}_{\omega}$ is holomorphic with respect to $\omega$ in $\mathbb{D}$. Furthermore, $\omega \neq 0$ is an eigenvalue of 30 ) if and only if 1 is an eigenvalue of $\mathcal{S}_{\omega}$ according to $[19 \mathrm{a})$. If every $\omega \in \mathbb{D} \backslash\{0\}$ fulfilled $[30\}$, then since $\mathbb{D}$ is open, there would be a sequence $\left\{z_{j}\right\}_{j \in \mathbb{N}} \in \mathbb{D}$ and $\left\{v_{j}\right\}_{j \in \mathbb{N}} \in \mathbb{V}$ with $\left\|v_{j}\right\|_{\mathbb{W}}=1$, such that $z_{j} \rightarrow 0$ when $j \rightarrow \infty$ and

$$
\left|\mathcal{E}_{z_{j}}\left(v_{j}, v_{j}\right)\right|=\left|z_{j}^{2}\left\langle v_{j}, v_{j}\right\rangle_{\mathbb{W}}\right| \leq\left|z_{j}^{2}\right|, \forall j \in \mathbb{N} .
$$

Now since $\mathcal{E}_{0}$ is hermitian and coercive (the family of operators $\left\{\mathcal{E}_{\omega}\right\}_{\omega \in \mathbb{D}}$ was supposed coercive from the beginning), thus for all $v \neq 0$, there exists $\lambda>0$ such that

$$
\frac{\mathcal{E}_{0}(v, v)}{\|v\|_{\mathbb{V}}} \geq \lambda
$$

so

$$
0<\lambda=\lambda\left\|v_{j}\right\|_{\mathbb{W}}^{2} \leq \lambda\left\|v_{j}\right\|_{\mathbb{V}}^{2} \leq \mathcal{E}_{0}\left(v_{j}, v_{j}\right), \forall j
$$


since $\mathbb{V}$ is embedded in $\mathbb{W}$. But then, using the fact that $\mathcal{E}_{\omega}$ is holomorphic and 31 .

$$
\mathcal{E}_{0}\left(v_{j}, v_{j}\right) \leq\left|\mathcal{E}_{0}\left(v_{j}, v_{j}\right)-\mathcal{E}_{z_{j}}\left(v_{j}, v_{j}\right)\right|+\left|\mathcal{E}_{z_{j}}\left(v_{j}, v_{j}\right)\right|=O\left(\left|z_{j}\right|\right)
$$

and we obtain a contradiction with 32 for $j$ large enough. The desired result finally stems from Theorem 3.1 .

We give an application of the above theorem to the 3D viscoelastic and UCLD plate vibration problems

Corollary 3.1. Suppose that the complex elastic tensors $A(\omega)$ and $D(\omega)$ in (5) and (7) respectively, are holomorphic with respect to $\omega$ for $\omega \in \mathbb{D} \subset \mathbb{C}$ bounded. Suppose also that $0 \in \mathbb{D}$ and $A(0), D(0)$ are hermitian positive definite, i.e. $A(0)=A(0)^{H}$ (being the symbol $H$ the conjugate transpose) and $\xi^{H} A(0) \xi>0, \forall \xi \in$ $\mathbb{C}^{d \times d}$, where $d=3$ (equivalently $D(0)$ with $d=2$ ). Then problems (5) and (7) possess only a finite number of eigenvalues in each compact subset of $\mathbb{D}$.

Proof. The result derives from Theorem 3.2 by considering

$$
\mathcal{E}_{\omega}(u, v)=\int_{\Omega} A(\omega) e(u): e(\bar{v}) d x, \mathbb{V}=\left\{v \in H^{1}(\Omega ; \mathbb{C})^{3}: v=0 \text { on } \Gamma_{D}\right\}, \mathbb{W}=L^{2}(\Omega ; \mathbb{C})^{3}
$$

in problem (5) and

$$
\mathcal{E}_{\omega}(\mathrm{w}, \zeta)=\int_{\mathcal{O}} D(\omega) \nabla^{2} \mathrm{w}: \nabla^{2} \bar{\zeta} d x, \mathbb{V}=\left\{\zeta \in H^{2}(\mathcal{O} ; \mathbb{C}): \zeta=0, \nabla \zeta=0 \text { on } \Gamma_{D}\right\}, \mathbb{W}=L^{2}(\mathcal{O} ; \mathbb{C})
$$

in problem (7).

\section{Sensitivity analysis of $\omega_{1}$ with respect to the shape $\Omega$}

In order to apply a gradient method to the minimization of (11), we dedicate this section to the study of the shape differentiability of $\omega_{1}$ and eigenvectors $\left(u_{1}, w_{1}\right)$, solutions of (5) and (7) respectively. Nevertheless all results remain valid for $k \geq 2$ by replacing $\left(\omega_{1}, u_{1}, \mathrm{w}_{1}\right)$ with $\left(\omega_{k}, u_{k}, \mathrm{w}_{k}\right)$.

Before going any further, we recall the classical notion of shape derivative which goes back, at least, to Hadamard (see the modern reference books [16, 31]). Here, we follow the approach of Murat and Simon [32], 33] . Starting from a smooth reference open set $\Omega$, we consider domains of the type

$$
\Omega_{\theta}=(\operatorname{Id}+\theta)(\Omega),
$$

where Id is the identity mapping from $\mathbb{R}^{d}$ into $\mathbb{R}^{d}$, and $\theta \in W^{1, \infty}\left(\mathbb{R}^{d} ; \mathbb{R}^{d}\right)$. It is well-known that, for sufficiently small $\theta,(\operatorname{Id}+\theta)$ is a diffeomorphism in $\mathbb{R}^{d}$. We denote as $\mathcal{C}(\Omega)$ the set of admissible shapes defined as $\left\{(\operatorname{Id}+\theta)(\Omega), \theta \in W^{1, \infty}\left(\mathbb{R}^{d} ; \mathbb{R}^{d}\right)\right\}$.

Definition 4.1 (Shape derivative). Let $J(\Omega)$ be a mapping from the set of admissible shapes $\mathcal{C}(\Omega)$ to $\mathbb{R}$. The shape derivative of $J(\Omega)$ at $\Omega$ is defined as the Fréchet derivative in $W^{1, \infty}\left(\mathbb{R}^{d} ; \mathbb{R}^{d}\right)$ at 0 of the mapping $\theta \rightarrow J((I d+\theta)(\Omega))$, i.e.,

$$
J((I d+\theta)(\Omega))=J(\Omega)+J^{\prime}(\Omega)(\theta)+o(\theta) \text { with } \lim _{\theta \rightarrow 0} \frac{|o(\theta)|}{|\theta|}=0,
$$

where $J^{\prime}(\Omega)$ is a continuous linear form on $W^{1, \infty}\left(\mathbb{R}^{d} ; \mathbb{R}^{d}\right)$.

In his research on elastic plates 34 in 1907, Hadamard showed how to obtain the shape derivative of a functional $J(\Omega)$ by considering normal perturbations of the boundary $\Omega$. This fundamental result was made rigorous later by Zolésio [35] in the so-called "structure theorem" which states that when $J(\Omega)$ and 
$\Omega$ are smooth enough, one may write the shape derivative of $J(\Omega)$ as an integral over $\partial \Omega$, i.e. there exists $\mathcal{V} \in L^{1}(\partial \Omega)$ such that

$$
J^{\prime}(\Omega)(\theta)=\int_{\partial \Omega}(\theta \cdot n) \mathcal{V} d s
$$

To fix ideas, we give two examples of shape derivative.

Lemma 4.1. Let $\Omega$ be a smooth bounded open set and $\phi(x) \in W^{1,1}\left(\mathbb{R}^{d}\right)$. Define

$$
J_{1}(\Omega)=\int_{\Omega} \phi(x) d x .
$$

Then $J_{1}$ is differentiable at $\Omega$ and

$$
J_{1}^{\prime}(\Omega)(\theta)=\int_{\partial \Omega} \theta(x) \cdot n(x) \phi(x) d s
$$

for any $\theta \in W^{1, \infty}\left(\mathbb{R}^{d} ; \mathbb{R}^{d}\right)$. For $\phi(x) \in W^{2,1}\left(\mathbb{R}^{d}\right)$ define

$$
J_{2}(\Omega)=\int_{\partial \Omega} \phi(x) d s .
$$

Then $J_{2}$ is differentiable at $\Omega$ and

$$
J_{2}^{\prime}(\Omega)(\theta)=\int_{\partial \Omega} \theta(x) \cdot n(x)\left(\frac{\partial \phi}{\partial n}+H \phi\right) d s
$$

for any $\theta \in W^{1, \infty}\left(\mathbb{R}^{d} ; \mathbb{R}^{d}\right)$, where $H$ is the mean curvature of $\partial \Omega$ defined by $H=$ divn

Before computing the shape derivatives of our damping problem, we introduce the definitions of the Lagrangian and Eulerian derivatives.

Definition 4.2. The function $u: \Omega \rightarrow u(\Omega) \in H^{1}(\Omega)$ admits a Lagrangian (or material) derivative $Y(\Omega)(\theta)$ at a given domain $\Omega$, provided that the application

$$
\theta \in W^{1, \infty}\left(\mathbb{R}^{d} ; \mathbb{R}^{d}\right) \rightarrow \tilde{u}_{\theta}(\Omega):=u((I d+\theta) \Omega) \circ(I d+\theta) \in H^{1}(\Omega) .
$$

is Fréchet differentiable at $\theta=0$. Then $Y\left(\Omega_{0}\right)(\theta)=\tilde{u}_{\theta}^{\prime}(\Omega)$.

Definition 4.3. The function $u: \Omega \rightarrow u(\Omega)$ admits a Eulerian derivative $u^{\prime}(\Omega)(\theta)$ at a given domain $\Omega$, if $u$ admits a Lagrangian derivative $Y(\Omega)(\theta)$ at $\Omega$ in the direction $\theta$ and $\nabla u(\Omega) \cdot \theta \in H^{1}(\Omega)$. In that case

$$
u^{\prime}(\Omega)(\theta)=Y(\Omega)(\theta)-\nabla u(\Omega) \cdot \theta .
$$

\subsection{Differentiability of $\omega_{1}$}

Theorem 4.1. Suppose $\Omega$ measurable and bounded and $\omega_{1}(\Omega)$ simple. Then the first eigenfrequency $\omega_{1}(\Omega)$, $u_{1}(\Omega)\left(\right.$ for $d=3$ ) and $\mathrm{w}_{1}(\Omega)$ (for $d=2$ ) are shape differentiable.

Proof. We give a sketch of proof since it corresponds to an adaptation of the result exhibited in [14] chapter 5, page 210. Also, as a matter of simplicity, we detail the proof argument only for $d=3$.

Define $u_{\theta}$ and $\omega(\theta)$ as the solutions of the problem (5) within $\Omega_{\theta}=(\operatorname{Id}+\theta) \Omega$, where $\theta \in W^{1, \infty}\left(\mathbb{R}^{3} ; \mathbb{R}^{3}\right)$. In other words,

$$
\begin{cases}-\operatorname{div}\left(A(\omega) e\left(u_{\theta}\right)\right)-\omega^{2} \rho u_{\theta} & =0 \text { in } \Omega_{\theta} \\ \int_{\Omega_{\theta}} \rho\left|u_{\theta}\right|^{2} d x-1 & =0\end{cases}
$$


Let us consider the operator $\mathcal{F}: W^{1, \infty}\left(\mathbb{R}^{3} ; \mathbb{R}^{3}\right) \times V \times \mathbb{C} \rightarrow H^{-1}\left(\Omega ; \mathbb{C}^{3}\right) \times \mathbb{R}$, with $V$ defined by (36), such that $\mathcal{F}(\theta, u, \omega)=0$ represents the transported problem 35 on $\Omega$. The main idea of the proof is to apply the implicit function theorem to $\mathcal{F}$ in order to show that there exist differentiable functions $\omega_{1}(\theta)$ and $u_{1}(\theta)$ at $\theta=0$, solutions of 35$)$. For that purpose, we need to prove that the differential operator $D_{v, \omega} F\left(0, u_{1}, \omega_{1}\right)$ given by

$$
D_{v, \omega} \mathcal{F}\left(0, u_{1}, \omega_{1}\right)(\tilde{v}, \tilde{\mu})=\left(-\operatorname{div}\left(A\left(\omega_{1}\right) e(\tilde{v})\right)-\omega_{1}^{2} \rho \tilde{v}-\tilde{\mu}\left(\operatorname{div}\left(\partial_{\omega} A\left(\omega_{1}\right) e\left(u_{1}\right)\right)+2 \omega_{1} \rho u_{1}\right), 2 \int_{\Omega} \rho \tilde{v} \cdot \bar{u}_{1} d x\right),
$$

for every $(\tilde{v}, \tilde{\mu}) \in V \times \mathbb{C}$ is an isomorphism (by virtue of the inverse function theorem in Banach spaces) from $V \times \mathbb{C}$ on $H^{-1}\left(\Omega ; \mathbb{C}^{3}\right) \times \mathbb{C}$. Since $D_{v, \mu} \mathcal{F}\left(0, u_{1}, \omega_{1}\right)$ is continuous, according to the Banach theorem, to prove that $D_{v, \mu} \mathcal{F}\left(0, u_{1}, \omega_{1}\right)$ is an isomorphism one simply needs to show that $D_{v, \mu} \mathcal{F}\left(0, u_{1}, \omega_{1}\right)$ is a bijection. Thus, given $(Z, \Lambda) \in H^{-1}\left(\Omega ; \mathbb{C}^{3}\right) \times \mathbb{C}$, we need to establish that the problem

$$
\begin{cases}-\operatorname{div}\left(A\left(\omega_{1}\right) e(\tilde{v})\right)-\omega_{1}^{2} \rho \tilde{v}-\tilde{\mu}\left(\operatorname{div}\left(\partial_{\omega} A\left(\omega_{1}\right) e\left(u_{1}\right)\right)+2 \omega_{1} \rho u_{1}\right) & =Z \text { in } \Omega \\ 2 \int_{\Omega} \rho \tilde{v} \cdot \bar{u}_{1} d x & =\Lambda\end{cases}
$$

has an unique solution $(\tilde{v}, \tilde{\mu}) \in V \times \mathbb{C}$. This is true thanks to the fact that for a fixed $\omega \in \mathbb{C}$ (in our case $\left.\omega=\omega_{1}\right)$, the same argument as that given in 14 can be applied, namely in a variational sens the operator

$$
(-\operatorname{div}(A(\omega) e(\cdot)))^{-1}: V^{\prime} \rightarrow V \subset V^{\prime}
$$

with $V^{\prime}$ the dual of $V$, is compact and thus the Fredholm alternative theorem can be applied to the operator

$$
-\operatorname{div}\left(A\left(\omega_{1}\right) e(\cdot)\right)-\omega_{1}^{2} \rho \operatorname{Id}(\cdot)
$$

whose kernel is of dimension one since we assume $\omega_{1}$ simple. Thus, in order to be unique the solution of the above system, the following orthogonality condition must stand

$$
\left\langle Z-\tilde{\mu}\left(\operatorname{div}\left(\partial_{\omega} A\left(\omega_{1}\right) e\left(u_{1}\right)\right)+2 \omega_{1} \rho u_{1}\right), u_{1}\right\rangle=0
$$

which determines uniquely the value of $\tilde{\mu}$. The uniqueness of $\tilde{v}$ stems from the decomposition $\tilde{v}=v_{0}+s u_{1}$, where $v_{0}$ is a particular solution and $s \in \mathbb{R}$. $s$ is uniquely determined by the second condition of the system.

\subsection{Shape derivative in the case of a $3 D$ viscoelastic structure}

Theorem 4.2. Define the space

$$
V=\left\{v \in H^{1}(\Omega ; \mathbb{C})^{3}: v=0 \text { on } \Gamma_{D}\right\}
$$

Let $\left(\omega_{1}, u_{1}\right) \in \mathbb{C} \times V$ be the solution of the eigenvalue problem (5) and $\omega_{1}$ simple. Also let $A^{H}\left(\omega_{1}\right)=\overline{A\left(\omega_{1}\right)^{T}}$ be the conjugate transpose tensor of $A\left(\omega_{1}\right)$ and introduce the adjoint eigenvector $p_{1} \in V$ solution of

$$
\int_{\Omega} A^{H}\left(\omega_{1}\right) e\left(p_{1}\right): e(\bar{v}) d x=\tilde{\omega}_{1}^{2} \int_{\Omega} \rho\left(p_{1} \cdot \bar{v}\right) d x, \quad \forall v \in V
$$

with $\int_{\Omega} \rho\left|p_{1}\right|^{2} d x=1$ and $\tilde{\omega}_{1}^{2}=\overline{\omega_{1}^{2}}$. Then:

$$
\omega_{1}^{\prime}(\Omega)(\theta)=-\frac{\int_{\partial \Omega} \theta \cdot n\left(\omega_{1}^{2} \rho\left(u_{1} \cdot \bar{p}_{1}\right)-A\left(\omega_{1}\right) e\left(u_{1}\right): e\left(\bar{p}_{1}\right)\right) d s}{\int_{\Omega}\left(2 \omega_{1} \rho\left(u_{1} \cdot \bar{p}_{1}\right)-\partial_{\omega} A\left(\omega_{1}\right) e\left(u_{1}\right): e\left(\bar{p}_{1}\right)\right) d x} .
$$


Proof. As mentioned in the previous section, the fact that $\omega_{1}$ is simple ensures that $\omega_{1}(\Omega)$ and $u_{1}(\Omega)$ are shape-differentiable. Thus we can differentiate with respect to the domain both sides of the variational formulation of $(5)$

$$
\int_{\Omega} A\left(\omega_{1}\right) e\left(u_{1}\right): e(\bar{v}) d x=\omega_{1}^{2} \int_{\Omega} \rho\left(u_{1} \cdot \bar{v}\right) d x, \quad \forall v \in V
$$

Taking $\theta \in W^{1, \infty}\left(\mathbb{R}^{3}, \mathbb{R}^{3}\right)$ with $\theta=0$ on $\Gamma_{N} \cup \Gamma_{D}$ :

$$
\begin{aligned}
& \int_{\partial \Omega} A\left(\omega_{1}\right) e\left(u_{1}\right): e(\bar{v})(\theta \cdot n) d s+\omega_{1}^{\prime}(\Omega)(\theta) \int_{\Omega}\left(\partial_{\omega} A\right) e\left(u_{1}\right): e(\bar{v}) d x+\int_{\Omega} A\left(\omega_{1}\right) e\left(u_{1}^{\prime}(\Omega)(\theta)\right): e(\bar{v}) d x \\
& =2 \omega_{1} \omega_{1}^{\prime}(\Omega)(\theta) \int_{\Omega} \rho\left(u_{1} \cdot \bar{v}\right) d x+\omega_{1}^{2} \int_{\Omega} \rho\left(u_{1}^{\prime}(\Omega)(\theta) \cdot \bar{v}\right) d x+\omega_{1}^{2} \int_{\partial \Omega} \rho\left(u_{1} \cdot \bar{v}\right)(\theta \cdot n) d s
\end{aligned}
$$

Applying the conjugate on both sides of equation (37) one gets

$$
\int_{\Omega} A\left(\omega_{1}\right) e(v): e\left(\bar{p}_{1}\right) d x=\omega_{1}^{2} \int_{\Omega} \rho\left(v \cdot \bar{p}_{1}\right) d x, \quad \forall v \in V,
$$

so taking $v=p_{1}$ in $(40)$ and $v=u_{1}^{\prime}(\Omega)(\theta)$ in 44 , one realizes that all the terms containing $u_{1}^{\prime}(\Omega)(\theta)$ in (40) cancel. Thus 40 yields

$$
\begin{aligned}
& \int_{\partial \Omega} A\left(\omega_{1}\right) e\left(u_{1}\right): e\left(\bar{p}_{1}\right)(\theta \cdot n) d s+\omega_{1}^{\prime}(\Omega)(\theta) \int_{\Omega}\left(\partial_{\omega} A\right) e\left(u_{1}\right): e\left(\bar{p}_{1}\right) d x \\
& =2 \omega_{1} \omega_{1}^{\prime}(\Omega)(\theta) \int_{\Omega} \rho\left(u_{1} \cdot \bar{p}_{1}\right) d x+\omega_{1}^{2} \int_{\partial \Omega} \rho\left(u_{1} \cdot \bar{p}_{1}\right)(\theta \cdot n) d s
\end{aligned}
$$

from which $(38)$ follows.

Remark 4.1. When the tensor $A$ is independent from the frequency $\omega$, we recover the well-known formula [19] for the derivative:

$$
\omega_{1}^{\prime}(\Omega)(\theta)=-\frac{\int_{\partial \Omega} \theta \cdot n\left(\omega_{1}^{2} \rho\left(u_{1} \cdot \bar{p}_{1}\right)-A\left(\omega_{1}\right) e\left(u_{1}\right): e\left(\bar{p}_{1}\right)\right) d s}{\int_{\Omega} 2 \omega_{1} \rho\left(u_{1} \cdot \bar{p}_{1}\right) d x}
$$

Furthermore if $A$ is real (since by definition is symmetric) then the problem becomes self-adjoint (i.e. $\left.p_{1}=u_{1}\right)$.

\subsection{Shape derivative of a composite sandwich structure}

We refer to the notations given in Section 2.2. Define a bounded working domain $\mathcal{O} \subset \mathbb{R}^{2}$ and an admissible shape $\Omega \subset \mathcal{O}$ occupied by a viscoelastic damping treatment. Within this framework, (7) can be formulated as a multi-phase problem. As mentioned in [12, a sharp interface between the different phases leads to numerical difficulties that can be avoided by considering a smeared or diffuse interface approach. We follow herein this smoothed formulation and as abuse of notation we consider the same eigenelements $\omega_{1}$ and $\mathrm{w}_{1}$. Let $D$ and $\rho$ be the smooth interpolations between $D_{g}, D_{p}$, (with respective surface densities $\left.\rho_{g}, \rho_{p}\right)$ occupying $\Omega$ and $\mathcal{O} \backslash \bar{\Omega}$ respectively, defined by

$$
\begin{array}{rlrl}
D=D_{g}-H_{\varepsilon}\left(d_{\Omega}\right) \Delta D, & \Delta D & :=\left(D_{g}-D_{p}\right), \\
\rho & =\rho_{g}-H_{\varepsilon}\left(d_{\Omega}\right) \Delta \rho, & \Delta \rho & :=\left(\rho_{g}-\rho_{p}\right),
\end{array}
$$

where $H_{\varepsilon}(r): \mathbb{R} \rightarrow[0,1]$ is a smooth approximation of the Heaviside function

$$
H_{\varepsilon}(r)=\left\{\begin{array}{ccc}
0 & \text { if } & r<-\varepsilon \\
\frac{1}{2}\left(1+\frac{r}{\varepsilon}+\frac{1}{\pi} \sin \left(\frac{\pi r}{\varepsilon}\right)\right) & \text { if } & -\varepsilon \leq r \leq \varepsilon \\
1 & \text { if } & r>\varepsilon
\end{array}\right.
$$


and $d_{\Omega}(x): \mathbb{R}^{d} \rightarrow \mathbb{R}$ the signed distance function at $x$ associated with $\Omega$

$$
d_{\Omega}(x)=\left\{\begin{array}{cl}
0 & \text { if } x \in \partial \Omega, \\
-\min _{x_{I} \in \partial \Omega}\left|x-x_{I}\right| & \text { if } x \in \Omega \\
\min _{x_{I} \in \partial \Omega}\left|x-x_{I}\right| & \text { if } x \in \mathcal{O} \backslash \bar{\Omega} .
\end{array}\right.
$$

Before giving the shape derivative of $\omega_{1}$ in the context of Section 2.2, we collect some general definitions and results related to the signed distance function $d_{\Omega}$ in $\mathbb{R}^{d}$ (for more details consult [12]).

Definition 4.4. Let $\Omega \subset \mathbb{R}^{d}$ be a Lipschitz bounded open set.

- For any $x \in \mathbb{R}^{d}$ we define the set of projections of $x$ on $\partial \Omega$ as

$$
\Pi_{\partial \Omega}(x)=\left\{y_{0} \in \partial \Omega \text { such that }\left|x-y_{0}\right|=\inf _{y \in \partial \Omega}|x-y|\right\} .
$$

When $\Pi_{\partial \Omega}(x)$ reduces to a single point, we will call it the projection $P_{\partial \Omega}(x)$ of $x$ onto $\partial \Omega$.

- The skeleton $\Sigma$ of $\partial \Omega$ is defined as

$$
\Sigma:=\left\{x \in \mathbb{R}^{d} \text { such that }\left(d_{\Omega}\right)^{2} \text { is not differentiable at } x\right\} .
$$

Lemma 4.2. Let $\Omega \subset \mathbb{R}^{d}$ be a Lipschitz bounded open set.

- A point $x \notin \partial \Omega$ has a unique projection $P_{\partial \Omega}(x)$ on $\partial \Omega$ if and only if $x \notin \Sigma$.

- $\Sigma$ has zero Lebesgue measure in $\mathbb{R}^{d}$.

The signed distance function is not, strictly speaking, shape differentiable in the sense of (33). One reason is the lack of smoothness of the gradient of $d_{\Omega}$ at the skeleton $\Sigma$. However, its pointwise values $d_{\Omega}(x)$ are shape differentiable for $x \in \mathcal{O} \backslash \Sigma$. In particular for $d=2$ (the result also holds for $d=3$ )

Proposition 4.1. Assume $\Omega \subset \mathcal{O}$ is an open set of class $C^{1}$, and fix a point $x \notin \Sigma$. Then $\theta \rightarrow d_{(I d+\theta) \Omega}(x)$ is Gâteaux-differentiable at $\theta=0$, as a mapping from $W^{1, \infty}\left(\mathcal{O} ; \mathbb{R}^{2}\right)$ into $\mathbb{R}$, and its derivative is

$$
d_{\Omega}^{\prime}(\theta)(x)=-\theta\left(P_{\partial \Omega}(x)\right) \cdot n\left(P_{\partial \Omega}(x)\right) .
$$

Theorem 4.3. Define the space $W=\left\{\zeta \in H^{2}(\mathcal{O} ; \mathbb{C}): \zeta=0, \nabla \zeta=0\right.$ on $\left.\Gamma_{D}\right\}$. Let $\left(\omega_{1}, \mathrm{w}_{1}\right) \in \mathbb{C} \times W$ be the solutions of the eigenvalue problem (7) and assume $\omega_{1}$ simple. Also let $D^{H}\left(\omega_{1}\right)=\overline{D\left(\omega_{1}\right)^{T}}$ be the conjugate transpose tensor of $D$ and introduce the adjoint eigenvector $q_{1} \in W$ solution of

$$
\int_{\mathcal{O}} D^{H}\left(\omega_{1}\right) \nabla^{2} q: \nabla^{2} \bar{\zeta} d x=\tilde{\omega}^{2} \int_{\mathcal{O}} \rho(q \cdot \bar{\zeta}) d x, \quad \forall \zeta \in W,
$$

with $\int_{\mathcal{O}} \rho\left|q_{1}\right|^{2} d x=1$ and $\tilde{\omega}_{1}^{2}=\overline{\omega_{1}^{2}}$. Then:

$$
\omega_{1}^{\prime}(\Omega)(\theta)=-\frac{\int_{\mathcal{O}} \partial_{r} H_{\epsilon}\left(d_{\Omega}\right)\left(\omega_{1}^{2} \Delta \rho\left(\mathrm{w}_{1} \cdot \bar{q}_{1}\right)-\Delta D\left(\omega_{1}\right) \nabla^{2} \mathrm{w}_{1}: \nabla^{2} \bar{q}_{1}\right) \theta\left(P_{\partial \Omega}(x)\right) \cdot n\left(P_{\partial \Omega}(x)\right) d x}{\int_{\mathcal{O}}\left(2 \omega_{1} \rho\left(\mathrm{w}_{1} \cdot \bar{q}_{1}\right)-\partial_{\omega} D\left(\omega_{1}\right) \nabla^{2} \mathrm{w}_{1}: \nabla^{2} \bar{q}_{1}\right) d x},
$$

where $P_{\partial \Omega}(x)$ is the projection of $x$ onto $\partial \Omega$. 
Proof. The shape differentiability of $\omega_{1}(\Omega)$ follows from the same argument as in the proof of Theorem 4.1 Hence we differentiate with respect to the domain both sides of the variational formulation of (5) but this time within the fixed domain $\mathcal{O}$

$$
\int_{\mathcal{O}} D\left(\omega_{1}\right) \nabla^{2} \mathrm{w}_{1}: \nabla^{2} \bar{\zeta} d x=\omega_{1}^{2} \int_{\mathcal{O}} \rho\left(\mathrm{w}_{1} \cdot \bar{\zeta}\right) d x, \quad \forall \zeta \in W
$$

Taking $\theta \in W^{1, \infty}\left(\mathbb{R}^{3}, \mathbb{R}^{3}\right)$ with $\theta=0$ on $\partial \mathcal{O}$

$$
\begin{aligned}
& \int_{\mathcal{O}} D^{\prime}(\Omega)(\theta)\left(\omega_{1}\right) \nabla^{2} \mathrm{w}_{1}: \nabla^{2} \bar{\zeta} d x+\omega_{1}^{\prime}(\Omega)(\theta) \int_{\mathcal{O}} \partial_{\omega} D \nabla^{2} \mathrm{w}_{1}: \nabla^{2} \bar{\zeta} d x+\int_{\mathcal{O}} D\left(\omega_{1}\right) \nabla^{2} \mathrm{w}_{1}^{\prime}(\Omega)(\theta): \nabla^{2} \bar{\zeta} d x \\
& =2 \omega_{1} \omega_{1}^{\prime}(\Omega)(\theta) \int_{\mathcal{O}} \rho\left(\mathrm{w}_{1} \cdot \bar{\zeta}\right) d x+\omega_{1}^{2} \int_{\mathcal{O}} \rho\left(\mathrm{w}_{1}^{\prime}(\Omega)(\theta) \cdot \bar{\zeta}\right) d x+\omega_{1}^{2} \int_{\mathcal{O}} \rho^{\prime}(\Omega)(\theta)\left(\mathrm{w}_{1} \cdot \bar{\zeta}\right) d x .
\end{aligned}
$$

Applying the same argument given in the proof of Theorem 4.2 by choosing the right test functions in 47) $\left(\zeta=q_{1}\right)$ and $45\left(\zeta=\mathrm{w}_{1}^{\prime}(\Omega)(\theta)\right)$, 47) yields

$$
\begin{aligned}
& \int_{\mathcal{O}} D^{\prime}(\Omega)(\theta)\left(\omega_{1}\right) \nabla^{2} \mathrm{w}_{1}: \nabla^{2} \bar{q}_{1} d x+\omega_{1}^{\prime}(\Omega)(\theta) \int_{\mathcal{O}} \partial_{\omega} D \nabla^{2} \mathrm{w}_{1}: \nabla^{2} \bar{q}_{1} d x \\
& =2 \omega_{1} \omega_{1}^{\prime}(\Omega)(\theta) \int_{\mathcal{O}} \rho\left(\mathrm{w}_{1} \cdot \bar{q}_{1}\right) d x+\omega_{1}^{2} \int_{\mathcal{O}} \rho^{\prime}(\Omega)(\theta)\left(\mathrm{w}_{1} \cdot \bar{q}_{1}\right) d x
\end{aligned}
$$

so

$$
\omega_{1}^{\prime}(\Omega)(\theta)=-\frac{\int_{\mathcal{O}}\left(\omega_{1}^{2} \rho^{\prime}(\Omega)(\theta)\left(\mathrm{w}_{1} \cdot \bar{q}_{1}\right)-D^{\prime}(\Omega)(\theta)\left(\omega_{1}\right) \nabla^{2} \mathrm{w}_{1}: \nabla^{2} \bar{q}_{1}\right) d x}{\int_{\mathcal{O}}\left(2 \omega_{1} \rho\left(\mathrm{w}_{1} \cdot \bar{q}_{1}\right)-\partial_{\omega} D\left(\omega_{1}\right) \nabla^{2} \mathrm{w}_{1}: \nabla^{2} \bar{q}_{1}\right) d x} .
$$

According to 42 , the shape derivatives $\rho^{\prime}(\Omega)(\theta)$ and $D^{\prime}(\Omega)(\theta)$ read

$$
\begin{array}{ll}
\rho^{\prime}(\Omega)(\theta)(x) & =-\partial_{r} H_{\epsilon}\left(d_{\Omega}\right) d_{\Omega}^{\prime}(\theta)(x) \Delta \rho \\
D^{\prime}(\Omega)(\theta)\left(x, \omega_{1}\right) & =-\partial_{r} H_{\epsilon}\left(d_{\Omega}\right) d_{\Omega}^{\prime}(\theta)(x) \Delta D\left(\omega_{1}\right)
\end{array}
$$

where $d_{\Omega}^{\prime}(\theta)(x)$ is given by equation 44 .

Obtaining a descent direction from 46 is not necessarily easy and we would prefer to recover the classical shape derivative structure of a surface integral on the interface.

Corollary 4.1. Suppose that the interface is roughly plane (i.e. the principal curvatures can be neglected) and the thickness parameter $\varepsilon$ of the diffuse interface is small. Then a good approximation of (46) is

$$
\omega_{1}^{\prime}(\Omega)(\theta) \approx-\frac{\int_{\Gamma} \theta \cdot n\left(\omega_{1}^{2} \Delta \rho\left(\mathrm{w}_{1} \cdot \bar{q}_{1}\right)-\Delta D\left(\omega_{1}\right) \nabla^{2} \mathrm{w}_{1}: \nabla^{2} \bar{q}_{1}\right) d s}{\int_{\mathcal{O}}\left(2 \omega_{1} \rho\left(\mathrm{w}_{1} \cdot \bar{q}_{1}\right)-\partial_{\omega} D\left(\omega_{1}\right) \nabla^{2} \mathrm{w}_{1}: \nabla^{2} \bar{q}_{1}\right) d x},
$$

where $\Gamma=\partial \Omega \cap \operatorname{int}(\mathcal{O})$.

For the proof consult [12].

\section{Level-set method for topology optimization}

From the previous sections we have all the necessary theoretical ingredients to introduce a gradient method for the minimization of an objective function $J(\Omega)$ (see in particular 33 for the definition of shape derivative). As stated in (34), the shape derivative $J^{\prime}(\Omega)(\theta)$ can be written as a surface integral on $\Omega$ where 
the function $\mathcal{V}(x)$ is given in $(38)$ and $(49)$. Supposing $\mathcal{V}$ regular enough so it can be naturally extended to $\mathbb{R}^{d}$, a descent direction is found by defining the vector field

$$
\theta=-\mathcal{V} n
$$

where $n$ is a natural extension of the normal (which we implicitly assume for the moment that exists). Then we update the shape $\Omega$ as

$$
\Omega_{t}=(I d+t \theta) \Omega,
$$

where $t>0$ is a small descent step. Formally we obtain

$$
J\left(\Omega_{t}\right)=J(\Omega)-t \int_{\partial \Omega} \mathcal{V}^{2} d s+O\left(t^{2}\right)
$$

which guarantees the decrease of the objective function. We remark that if $\mathcal{V}$ turns out to be not regular enough (as it is the case for some objective functions) there are other possible choices for the definition of the descent direction [36].

First introduced in [13, the level-set method is a technique for capturing interfaces which are implicitly defined by the zero level-set of an auxiliary function. In particular, this method has been successfully applied to topology optimization problems $\left[37,18,17\right.$. Let the bounded domain $\mathcal{O} \subset \mathbb{R}^{d}$ be the working domain in which all admissible shapes $\Omega$ are included. In numerical practice, the domain $\mathcal{O}$ will be meshed once and for all. We represent the boundary of $\Omega$ by means of a level-set function $\psi$ defined over $\mathcal{O}$ such that

$$
\begin{cases}\psi(x)=0 & \text { if } x \in \partial \Omega, \\ \psi(x)<0 & \text { if } x \in \Omega, \\ \psi(x)>0 & \text { if } x \in(\mathcal{O} \backslash \bar{\Omega}) .\end{cases}
$$

Under the action of a normal vector field $-\mathcal{V}(t, x) n(x)$, where $n(x)$ is a natural extension of the normal given by $[38$ ]

$$
n(x)=\frac{\nabla \psi}{|\nabla \psi|},
$$

the shape $\Omega$ evolves according to the Hamilton-Jacobi equation

$$
\frac{\partial \psi}{\partial t}(t, x)-\mathcal{V}(t, x)|\nabla \psi(t, x)|=0, \quad \forall t, \forall x \in \mathcal{O} .
$$

Equation (51) is posed in the whole reference domain $\mathcal{O}$, and not only on the boundary $\partial \Omega$, if the velocity $\mathcal{V}$ is known everywhere (as will be the case in the sequel). Transporting $\psi$ by (51) is analogous to moving the boundary of $\partial \Omega$ (the zero level-set of $\psi$ ) along the direction $\mathcal{V} n$.

A common choice of boundary condition for 51 is

$$
\frac{\partial \psi}{\partial n}=0 \text { on } \partial \mathcal{O} \text {. }
$$

As mentioned in [19, this boundary condition is easy to implement since there is no fixed value to assign for $\psi$ at the boundary, and it also allows the solution of (51) to satisfy a maximum principle. More precisely, new inclusions (or holes) in $\Omega$ can appear only by advecting the zero level-set of $\psi$ which changes its topology and cannot come from outside the domain $\mathcal{O}$ because of spurious negative values created by the boundary conditions.

The numerical solution of (51) is computed with a second order explicit upwind scheme 39] on a Cartesian grid. Since this scheme is explicit in time, the time stepping must satisfy a CFL condition.

Because of the advection process or numerical diffusion, the level-set function may become too flat or too steep leading to large errors either in the location of its zero level set or in the evaluation of its gradient by finite differences. Therefore, it is usual to regularize it periodically by solving the following problem

$$
\left\{\begin{aligned}
\frac{\partial \psi}{\partial t}(t, x)+\operatorname{sign}\left(\psi_{0}\right)(|\nabla \psi(t, x)|-1) & =0, \quad \forall t, \forall x \in \mathcal{O} \\
\psi(t=0, x) & =\psi_{0}(x) \quad \forall x \in \mathcal{O},
\end{aligned}\right.
$$

which admits as a stationary solution the signed distance to the initial interface $\left\{\psi_{0}(x)=0\right\}$ [17. 


\section{Numerical analysis}

\subsection{Material properties}

Within this section we explain how to In order to extend the state equation (5) to the whole domain $\mathcal{O}$, we use the same "ersatz material" approach as [17. This approach amounts to filling the holes $\mathcal{O} \backslash \Omega$ by a weak phase mimicking void but avoiding the singularity of the rigidity matrix. This approach in needed in our $3 \mathrm{D}$ framework but not in the $2 \mathrm{D}$ since this is rather a multi-phase problem. We define an elasticity tensor $A^{\star}(x)$, which is a mixture of $A$ in $\Omega$ and of the weak material mimicking holes in $\mathcal{O} \backslash \Omega$, as

$$
A^{\star}(x)=\chi_{A}(x) A, \quad \chi_{A}(x)= \begin{cases}1, & \text { if } x \in \Omega, \\ \delta_{A}, & \text { if } x \in \mathcal{O} \backslash \Omega .\end{cases}
$$

We also need to apply the same procedure for the material density by introducing a mixture density

$$
\rho^{\star}(x)=\chi_{\rho}(x) \rho, \quad \chi_{\rho}(x)= \begin{cases}1 & \text { if } x \in \Omega \\ \delta_{\rho} & \text { if } x \in \mathcal{O} \backslash \Omega .\end{cases}
$$

For eigenfrequency optimization making a correct choice for the threshold parameters $\delta_{A}$ and $\delta_{\rho}$ is always delicate since a bad combination can yield spurious eigenmodes localized in the ersatz material [19].

In the case of the composite sandwich structure equation (7), the tensor $D$ and the density $\rho$ are evaluated according to the smooth multi-phase approximation (42) considering $d_{\Omega}=\psi$, i.e. the current level-set function. Indeed the level-set function $\psi$ is periodically reinitialized through (52). We set $\varepsilon=1.5 \Delta x$ in (43) where $\Delta x$ is the characteristic size of the computational mesh. We remark that in the multi-phase framework we do not need an ersatz material since the whole domain $\mathcal{O}$ is covered by the base plate material which makes the rigidity matrix of the composite sandwich always non-singular, no matter the shape of the damping layer.

\subsection{Solving the discrete non-linear eigenvalue problem}

Denote as

$$
\mathcal{T}_{h}(\omega) u_{h}=0, \quad u_{h} \in V_{h}
$$

the matrix representation of the discretization of problem (16) that stems from the weak (or variational) formulation of (5) (the space $V_{h}$ represents the finite element approximation space of (36)). Numerous numerical methods such as the modal strain energy method [40] and the asymptotic numerical method [4]. have been developed to solve the resulting nonlinear problem. An overview of the different methods of resolution can be found in [42, 43, 44. In our case we simply opt for applying the Newton's method to the extended system

$$
F_{z}\left(\omega, u_{h}\right)=\left(\begin{array}{c}
\mathcal{T}_{h}(\omega) \cdot u_{h} \\
\bar{z} \cdot u_{h}-1
\end{array}\right)=0
$$

where $z \in V_{h}$ is an arbitrary vector, fixed once and for all, such that $\|z\|=1$ and $\bar{z} \cdot u_{h}\left(\omega^{*}\right) \neq 0$ with $u_{h}\left(\omega^{*}\right)$ the exact eigenvector of (55) for eigenvalue $\omega^{*}$. Hence the second equation in (56) represents a normalization condition on $u_{h}$. The numerical solution $\left(\omega_{1}^{\ell}, u_{1}^{\ell}\right)$ obtained by FEM discretization of the problem (15) is chosen as the initial value $\left(\omega^{0}, u_{h}^{0}\right)$ and $z=u_{h}^{0}$. The Newton equation of (56)

$$
F_{z}\left(\omega^{n}, u_{h}^{n}\right)+\partial F_{z}\left(\omega^{n}, u_{h}^{n}\right)\left(\begin{array}{c}
\omega^{n+1}-\omega^{n} \\
u_{h}^{n+1}-u_{h}^{n}
\end{array}\right)=0
$$

gives the following update rules:

$$
\begin{gathered}
\omega^{n+1}=\omega^{n}-\frac{1}{\bar{z} \cdot \mathcal{T}_{h}^{-1}\left(\omega^{n}\right) \cdot \mathcal{T}_{h}^{\prime}\left(\omega^{n}\right) \cdot u_{h}^{n}}, \\
u_{h}^{n+1}=\left(\omega^{n}-\omega^{n+1}\right) \mathcal{T}_{h}^{-1}\left(\omega^{n}\right) \cdot \mathcal{T}_{h}^{\prime}\left(\omega^{n}\right) \cdot u_{h}^{n},
\end{gathered}
$$

where the matrices $\mathcal{T}_{h}^{-1}\left(\omega^{n}\right)$ and $\mathcal{T}_{h}^{\prime}\left(\omega^{n}\right)$ respectively stand for the inverse and the derivative with respect to $\omega$ of the FEM matrix $\mathcal{T}_{h}\left(\omega^{n}\right)$. We iterate until $\left|\omega^{n+1}-\omega^{n}\right| /\left|\omega^{n}\right|<$ tol with $t o l<<1$. 


\subsection{Computing a descent direction}

The gradient of the loss factor

$$
\eta(\Omega)=\frac{\operatorname{Im}\left(\omega_{1}^{2}\right)}{\operatorname{Re}\left(\omega_{1}^{2}\right)}
$$

can be derived from the formulas given in Sections 4.2 and 4.3 . Applying the product rule

$$
\begin{aligned}
\eta^{\prime}(\Omega)(\theta) & =2 \frac{\operatorname{Re}\left(\omega_{1}^{2}\right) \operatorname{Im}\left(\omega_{1} \omega_{1}^{\prime}(\theta)(\Omega)(\theta)\right)-\operatorname{Im}\left(\omega_{1}^{2}\right) \operatorname{Re}\left(\omega_{1} \omega_{1}^{\prime}(\Omega)(\theta)\right)}{\operatorname{Re}\left(\omega_{1}^{2}\right)^{2}}=2 \frac{\operatorname{Im}\left(\overline{\omega_{1}^{2}} \omega_{1} \omega_{1}^{\prime}(\Omega)(\theta)\right)}{\operatorname{Re}\left(\omega_{1}^{2}\right)^{2}} \\
& =2 \frac{\operatorname{Im}\left((1-i \eta) \omega_{1} \omega_{1}^{\prime}(\Omega)(\theta)\right)}{\operatorname{Re}\left(\omega_{1}^{2}\right)} .
\end{aligned}
$$

\subsubsection{D viscoelastic structure}

For a given $\omega_{1}$, define the constant

$$
\beta\left(\omega_{1}\right):=\int_{\Omega}\left(2 \omega_{1} \rho\left(u_{1} \cdot \bar{p}_{1}\right)-\partial_{\omega} A\left(\omega_{1}\right) e\left(u_{1}\right): e\left(\bar{p}_{1}\right)\right) d x
$$

and the function

$$
\gamma\left(\omega_{1}\right)(x):=\omega_{1}^{2} \rho\left(u_{1} \cdot \bar{p}_{1}\right)-A\left(\omega_{1}\right) e\left(u_{1}\right): e\left(\bar{p}_{1}\right) .
$$

Write (38) as

$$
\omega_{1}^{\prime}(\Omega)(\theta)=-\frac{\int_{\partial \Omega} \theta \cdot n\left(\overline{\beta\left(\omega_{1}\right)} \gamma\left(\omega_{1}\right)\right) d s}{\left|\beta\left(\omega_{1}\right)\right|^{2}} .
$$

Then since $\theta$ and the normal $n$ are real-valued, (57) reads

$$
\eta^{\prime}(\Omega)(\theta)=-\frac{2}{\operatorname{Re}\left(\omega_{1}^{2}\right)\left|\beta\left(\omega_{1}\right)\right|^{2}} \int_{\partial \Omega}(\theta \cdot n) \operatorname{Im}\left((1-i \eta) \omega_{1} \overline{\beta\left(\omega_{1}\right)} \gamma\left(\omega_{1}\right)\right) d s .
$$

The above expression provides directly a normal descent direction $\theta=V n$, with

$$
V=\frac{2}{\operatorname{Re}\left(\omega_{1}^{2}\right)\left|\beta\left(\omega_{1}\right)\right|^{2}} \operatorname{Im}\left((1-i \eta) \omega_{1} \overline{\beta\left(\omega_{1}\right)} \gamma\left(\omega_{1}\right)\right) .
$$

\subsubsection{Composite sandwich structure}

Following the same development, with the definitions

$$
\beta\left(\omega_{1}\right):=\int_{\mathcal{O}}\left(2 \omega_{1} \rho\left(\mathrm{w}_{1} \cdot \bar{q}_{1}\right)-\partial_{\omega} D\left(\omega_{1}\right) \nabla^{2} \mathrm{w}_{1}: \nabla^{2} \bar{q}_{1}\right) d x
$$

and

$$
\gamma\left(\omega_{1}\right)(x):=\omega_{1}^{2}\left(\rho_{g}-\rho_{c}\right)\left(\mathrm{w}_{1} \cdot \bar{q}_{1}\right)-\left(D_{g}-D_{c}\right)\left(\omega_{1}\right) \nabla^{2} \mathrm{w}_{1}: \nabla^{2} \bar{q}_{1},
$$

the same shape derivative (58) applies. 


\subsection{Test cases}

Numerical test cases involving the structure eigenvalues in topology optimization are usually not wellposed since less structure implies a higher eigenfrequency as a result of the ersatz material. Hence the optimizer converges to a trivial solution without any material. Typical remedies to solve this classical issue are including non-structural masses, defining the problem as a reinforcement problem or imposing a mass equality constraint (consult for instance [21]). For the following test cases we apply the first two solutions.

The 3D and 2D eigenvalue computations are performed using Freefem $++[45$, and the 3D results are rendered with XD3D [46.

The chosen viscoelastic properties for the 3D and 2D cases satisfy the holomorphic condition stated in Section 3 (except on a finite number of poles). That being said, the optimization process remains valid for any other viscoelastic material for which we can assume that the spectrum is discrete. Otherwise, as explained earlier, numerical difficulties, such as spurious eigenvalues, arise when dealing with a continuous spectrum or an accumulation point in the spectrum [47.

Remark 6.1. Given a certain operator $\mathbb{S}: V \rightarrow V$ (where $V$ is an adequate function space) and a sequence of discretized operators $\mathbb{S}_{n}: V_{n} \rightarrow V_{n}$ with $\mathbb{S}_{n} \rightarrow \mathbb{S}$ in the operator norm when $n \rightarrow \infty\left(V_{n}\right.$ is for instance a finite element subspace of $V$ ), we say that the eigenvalues $\omega_{n}$ are spurious in the sense that

$$
\omega_{n} \in \operatorname{Spectrum}\left(\mathbb{S}_{n}\right), \quad \omega_{n} \rightarrow \omega_{\infty} \notin \operatorname{Spectrum}(\mathbb{S}) .
$$

\subsubsection{D viscoelastic structure}

We optimize a three-dimensional cantilever. The working domain $\mathcal{O}$ is of size $1 \mathrm{~m} \times 2 \mathrm{~m} \times 1 \mathrm{~m}$ (discretized with a $20 \times 40 \times 20$ mesh). The viscoelastic material corresponds to 3 M ISD112 with complex Young modulus

$$
E_{c}(\omega)=\left(1+\nu_{c}\right) G_{0}\left(1+\sum_{k=1}^{3} \frac{\Delta_{k} \omega}{\omega-i z_{k}}\right), G_{0}=0.5 \mathrm{MPa},
$$

density $\rho_{c}^{V}=1600 \mathrm{~kg} / \mathrm{m}^{3}$ and Poisson coefficient $\nu_{c}=0.5$ (consult [48] for the values of the not listed constants $z_{k}$ and $\Delta_{k}$ ). A zero displacement boundary condition is imposed on the left side of the domain and four cubic cells on the middle of the right side (heavy tip mass with a material density 100 times heavier than the viscoelastic material) are not subject to optimization (see Figures 4 and 5). As mentioned before, this is one of the possible solutions to avoid a trivial optimal shape made only of ersatz material. Indeed, since the ersatz material has by definition a very low density (thus mass), the weight of the structure is minimized while keeping the same eigenfrequency by puling out all the material from the design space. The heavy tip mass is fixed during the optimization process so if the algorithm tried to pull out all the rest of the material from the box, the structure would break (the heavy mass must be connected to the fixed boundary condition). The ersatz material is characterized by the same Poisson's ratio as the viscoelastic material, a smaller Young's modulus by a factor $\delta_{A}=10^{-2}$ and a smaller density by a factor $\delta_{\rho}=10^{-4}$ (see (53) and (54)). The objective function is given as a linear combination of the negative loss factor (since we minimize) and the volume of the structure

$$
J(\Omega)=-\eta+\ell \frac{|\Omega|}{|\mathcal{O}|},
$$

where $\ell=0.01$ is a fixed Lagrange multiplier for the volume constraint. The heavy tip mass is positioned on the rectangular (and not the square) face of the design domain in order to avoid the symmetric bending modes on the $X$ and $Y$ axis. This condition is not necessarily sufficient to enforce the first eigenvalue $\omega_{1}$ to be simple (our computations are based on this assumption) so we check at each iteration the existence of multiple eigenvectors. The modal loss factors $\eta$ of the initial and optimal shapes are respectively 0.025 and 0.041 . 
Remark 6.2. In this case $\Omega$ corresponds to a uniform isotropic viscoelastic structure such that

$$
A(\omega)=f(\omega) A_{0}
$$

where $f$ is a scalar function and $A_{0}$ is a real isotropic material independent of $\omega$ (both defined accordingly to equation (59) ). Hence solving the problem (5) is equivalent to solving the equation:

$$
-\operatorname{div}\left(A_{0} e(u)\right)=\lambda \rho u \text { in } \Omega,
$$

which has a countably infinite family of solutions $\left(\lambda_{k}, u_{k}\right)_{k \geq 1}$, labeled by increasing order of the eigenfrequency. The eigenmodes $u_{k}$ coincide with the eigenmodes of the original viscoelastic problem and we can easily check that $p_{k}=u_{k}$, so the optimization problem is self-adjoint. In order to compute the modal loss factor $\eta$, we determine the original eigenvalue $\omega_{1}$ as the solution of the non-linear scalar equation:

$$
\omega^{2}-f(\omega) \lambda_{1}=0,
$$

via Newton's method with the initial value $\omega_{1}^{0}=\lambda_{1}^{1 / 2}$.
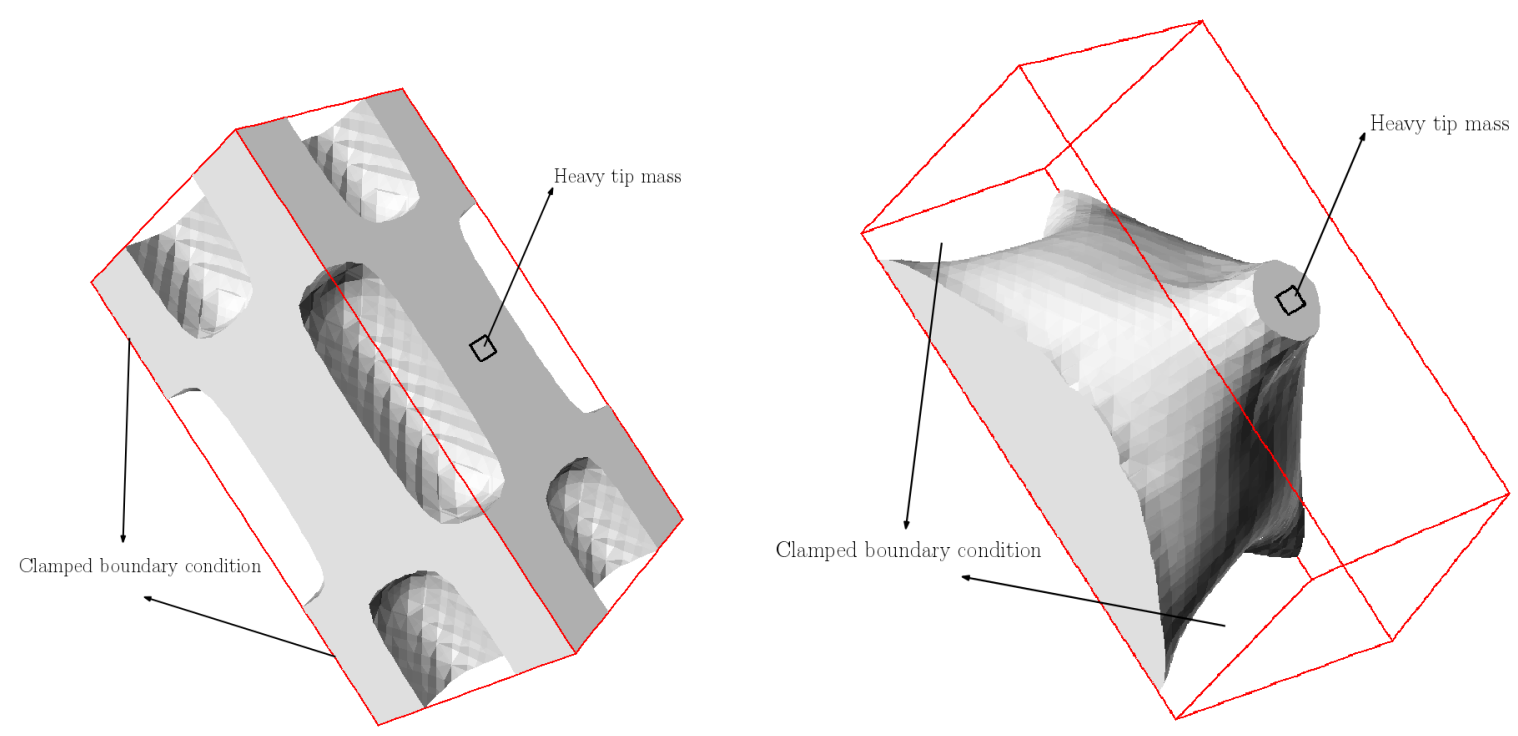

Figure 4: Initial (with a cavity inside) and optimized shapes of a 3D cantilever.

\subsubsection{Composite sandwich structure}

Now we optimize a square plate $\mathcal{O}$ with all edges clamped $(\mathrm{w}=\partial \mathrm{w} / \partial n=0$ on $\partial \mathcal{O})$. The width and the length of the working domain are both $400 \mathrm{~mm}$, discretized with a $40 \times 40$ mesh. The thickness of the base plate and the damping layer are $8.75 \mathrm{~mm}$ and $1.25 \mathrm{~mm}$, respectively. The base layer is made of aluminum whose Young's modulus is $E_{p}=69 \mathrm{GPa}$, Poisson's coefficient $\nu_{p}=0.3$ and volumetric density $\rho_{p}^{V}=2760 \mathrm{~kg} / \mathrm{m}^{3}$. The viscoelastic coating corresponds to LD-400, a fractional derivative model material depending on the temperature $T$ with complex Young modulus

$$
E_{c}(\omega)=\frac{a_{0}+a_{1}(i \omega \alpha(T))^{\beta}}{1+c_{1}(i \omega \alpha(T))^{\beta}} \mathrm{MPa},
$$




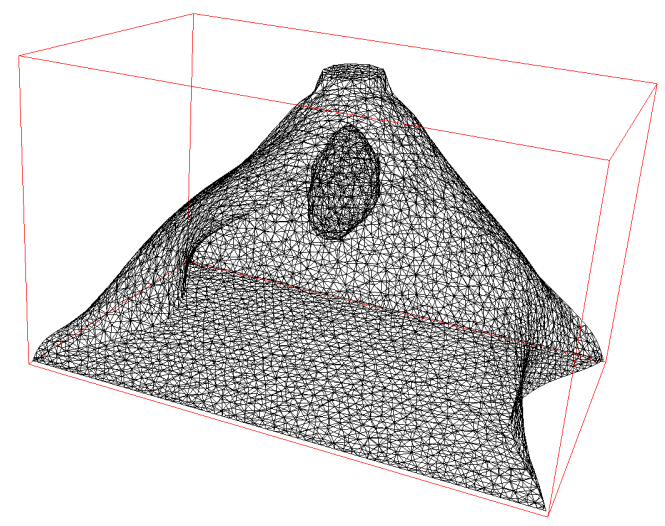

Figure 5: The optimized shape possesses a small inner cavity. If the hole is filled with the viscoelastic material, the value of $\eta$ remains almost constant (actually it slightly diminishes) so the value of the composite objective function 60 increases. The authors verified that the inner cavity is not present when the whole working domain $\mathcal{O}$ is used as initial shape in the optimization process.

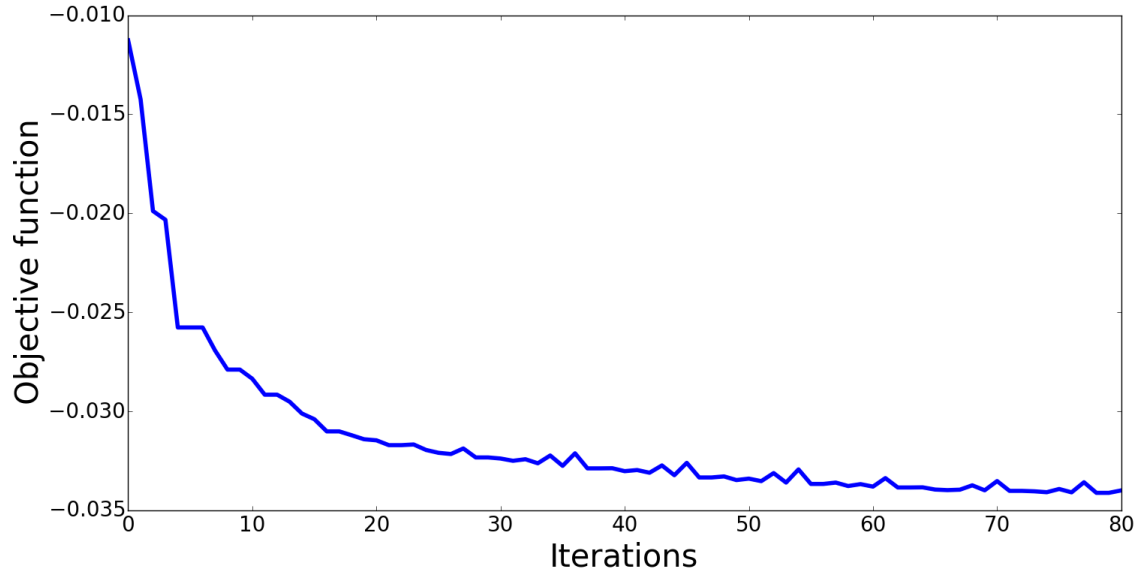

Figure 6: Convergence history for the optimization of the 3D cantilever. 
density $\rho_{c}^{V}=1524 \mathrm{~kg} / \mathrm{m}^{3}$ and Poisson coefficient $\nu_{c}=0.5$. The four parameters $a_{0}=332.2, a_{1}=2485.2, c_{1}=$ $0.12, \beta=0.47$ and the shift factor $\alpha(T)$ are available in [22. In our case we will consider the temperature $T$ to be constant and equal to $27^{\circ} \mathrm{C}$.

The objective function $J(\Omega)$ is the same as $(60)$ but this time with $\ell=10^{-1}$. The modal loss factors of the initial and optimized shapes are respectively $1.5 \times 10^{-2}$ and $2.31 \times 10^{-2}$ (see Figure 7). We remark that the optimized shape of the viscoelastic treatment coincides with the one obtained in [2] using the SIMP method. The result may not be completely intuitive at first since a significant portion of the damping reinforcement material lays outside the eigenmode largest deflection zone (Figure 8). However, as explained in Section 2.2, the sandwich energy is mainly dissipated as a result of in-plane compression and extension of the damping material under the flexural stress of the base plate. Hence the optimal damping reinforcement material layout and the area with the largest extensional strain energy density overlap (see Figure 9).
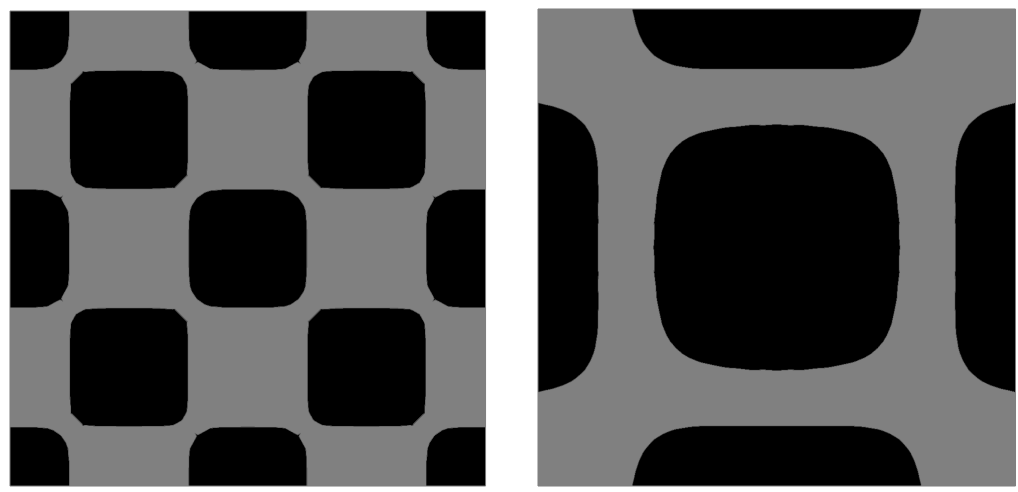

Figure 7: Initial and optimized shapes of the composite plate. The aluminum phase is shown in gray and the (superposed) viscoelastic one in black.

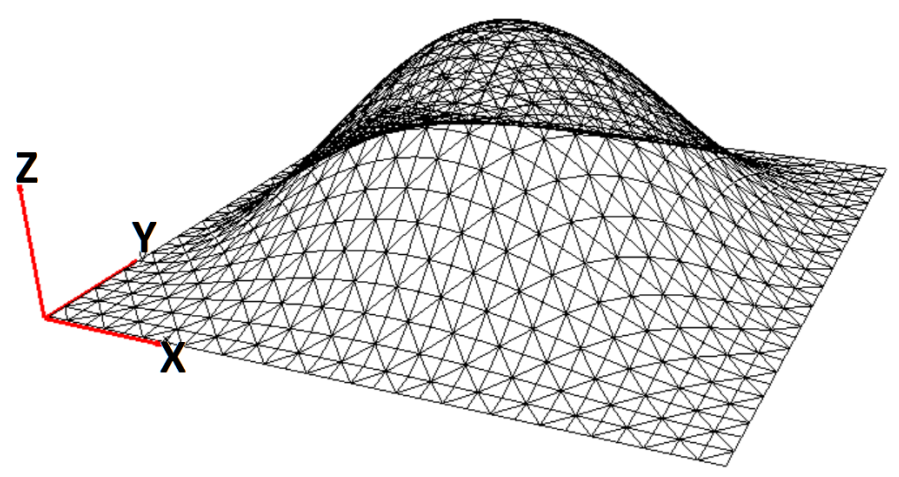

Figure 8: Real part of the eigenmode $w_{1}$ for the final shape. 


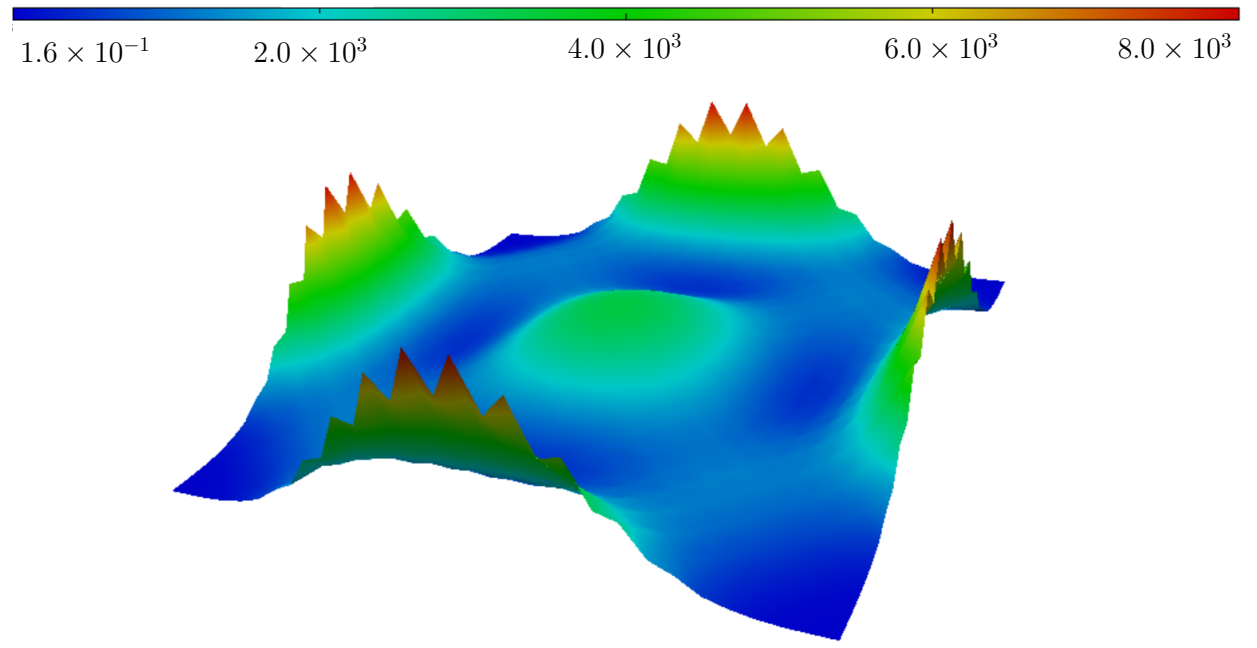

Figure 9: In-plane extensional strain energy density $\left(\mathrm{J} / \mathrm{m}^{2}\right)$.

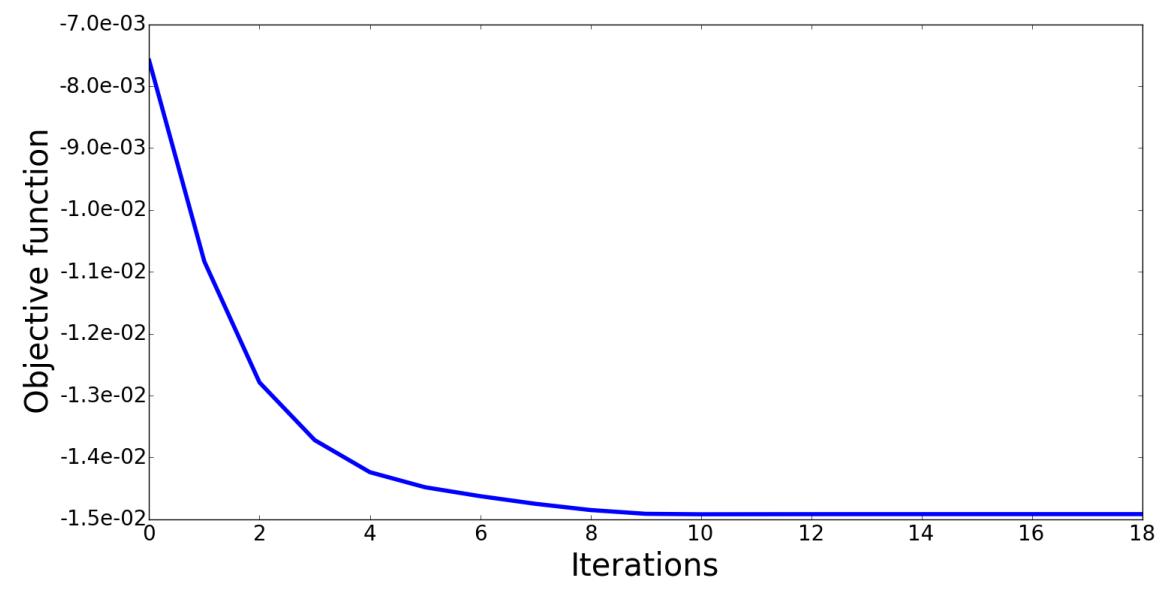

Figure 10: Convergence history for the optimization of the 2D composite sandwich plate. 


\section{Acknowledgments}

This research work was carried out in the framework of IRT SystemX, Paris-Saclay, France, and therefore supported with public funds within the scope of the French Program "Investissements d'Avenir". The authors also thank the financial support of the industrial partners of the TOP project: Safran, Renault, Airbus Group and ESI Group. We would like additionally to show our gratitude to Julien Cortial who read carefully this article providing a very useful feedback and Prof. El Mostafa Daya for fruitful discussions and kind assistance on viscoelastic sandwich modeling. 


\section{References}

\section{References}

[1] Z. Ling, X. Ronglu, W. Yi, A. El-Sabbagh, Topology optimization of constrained layer damping on plates using Method of Moving Asymptote (MMA) approach, Shock and Vibration 18 (1-2) (2011) 221-244. doi:\{10.3233/SAV-2010-0583\}

[2] S. Y. Kim, Topology design optimization for vibration reduction: reducible design variable method, Ph.D. thesis, Queen's University (2011).

[3] W. Zheng, Y. Lei, S. Li, Q. Huang, Topology optimization of passive constrained layer damping with partial coverage on plate, Shock and Vibration 20 (2) (2013) 199-211. doi:\{10.3233/SAV-2012-00738\}

[4] S. Y. Kim, C. K. Mechefske, I. Y. Kim, Optimal damping layout in a shell structure using topology optimization, Journal of Sound and Vibration 332 (12) (2013) 2873-2883. doi:http://dx.doi.org/10.1016/j.jsv.2013.01.029 URL http://www.sciencedirect.com/science/article/pii/S0022460X13000771

[5] A. El-Sabbagh, A. Baz, Topology optimization of unconstrained damping treatments for plates, Engineering Optimization 46 (9) (2014) 1153-1168. doi:\{10.1080/0305215X.2013.832235\}

[6] M. Bendsøe, Methods for optimization of structural topology, shape and material, Springer Verlag, New York, 1995.

[7] Z. Fang, L. Zheng, Topology Optimization for Minimizing the Resonant Response of Plates with Constrained Layer Damping Treatment, Shock and Vibration 2015. doi:\{10.1155/2015/376854\}

[8] K. A. James, H. Waisman, Topology optimization of viscoelastic structures using a time-dependent adjoint method Computer Methods in Applied Mechanics and Engineering 285 (2015) 166-187. doi:http://dx.doi.org/10.1016/j.cma. 2014.11 .012 URL http://www.sciencedirect.com/science/article/pii/S004578251400437X

[9] K.-S. Yun, S.-K. Youn, Multi-material topology optimization of viscoelastically damped structures under time-dependent loading, Finite Elements in Analysis and Design 123 (2017) 9-18. doi:http://dx.doi.org/10.1016/j.finel.2016.09.006 URL http://www.sciencedirect.com/science/article/pii/S0168874X16303572

[10] M. Ansari, A. Khajepour, E. Esmailzadeh, Application of level set method to optimal vibration control of plate structures Journal of Sound and Vibration 332 (4) (2013) 687-700. doi:http://dx.doi.org/10.1016/j.jsv.2012.09.006 URL http://www.sciencedirect.com/science/article/pii/S0022460X12006955

[11] M. van der Kolk, G. J. van der Veen, J. de Vreugd, M. Langelaar, Multi-material topology optimization of viscoelastically damped structures using a parametric level set method, Journal of Vibration and Control.

[12] G. Allaire, C. Dapogny, G. Delgado, G. Michailidis, Multi-phase structural optimization via a level set method, ESAIM: Control, Optimisation and Calculus of Variations 20 (2) (2014) 576-611.

[13] S. Osher, J. A. Sethian, Fronts propagating with curvature-dependent speed: algorithms based on Hamilton-Jacobi formulations, Journal of Computational Physics 79 (1) (1988) 12-49.

[14] A. Henrot, M. Pierre, Variation et optimisation de formes: une analyse géométrique, Vol. 48, Springer Science \& Business Media, 2006.

[15] F. Murat, J. Simon, Quelques résultats sur le contrôle par un domaine géométrique, VI Laboratoire d'Analyse Numérique, 1974.

[16] O. Pironneau, Optimal shape design for elliptic systems, Springer Science \& Business Media, 2012.

[17] G. Allaire, F. Jouve, A.-M. Toader, Structural optimization using sensitivity analysis and a level-set method, Journal of Computational Physics 194 (1) (2004) 363-393.

[18] M. Y. Wang, X. Wang, D. Guo, A level set method for structural topology optimization, Computer Methods in Applied Mechanics and Engineering 192 (1) (2003) 227-246.

[19] G. Allaire, F. Jouve, A level-set method for vibration and multiple loads structural optimization, Computer Methods in Applied Mechanics and Engineering 194 (30) (2005) 3269-3290.

[20] G. Allaire, Shape optimization by the homogenization method, Vol. 146, Springer Science \& Business Media, 2012.

[21] M. P. Bendsøe, Topology optimization: theory, methods and applications, Springer, 2003.

[22] D. I. Jones, Handbook of viscoelastic vibration damping, John Wiley \& Sons, 2001.

[23] W. N. Findley, F. A. Davis, Creep and relaxation of nonlinear viscoelastic materials, Courier Corporation, 2013.

[24] J. N. Reddy, Mechanics of laminated composite plates: theory and analysis, CRC press, 1997.

[25] G. Parthasarathy, C. Reddy, N. Ganesan, Partial coverage of rectangular plates by unconstrained layer damping treatments, Journal of Sound and Vibration 102 (2) (1985) 203-216.

[26] M. Carfagni, E. Lenzi, M. Pierini, The loss factor as a measure of mechanical damping, in: SPIE proceedings series, Society of Photo-Optical Instrumentation Engineers, 1998, pp. 580-584.

[27] L. Ambrosio, G. Buttazzo, An optimal design problem with perimeter penalization, Calculus of Variations and Partial Differential Equations 1 (1) (1993) 55-69.

[28] A. Chambolle, A density result in two-dimensional linearized elasticity, and applications, Archive for rational mechanics and analysis 167 (3) (2003) 211-233.

[29] D. Chenais, On the existence of a solution in a domain identification problem, Journal of Mathematical Analysis and Applications 52 (2) (1975) 189-219.

[30] T. Kato, Perturbation theory for linear operators, Vol. 132, Springer Science \& Business Media, 2013.

[31] J. Sokołowski, J. Zolesio, Introduction to shape optimization: Shape sensitivity analysis. 1992.

[32] F. Murat, J. Simon, Etude de problèmes d'optimal design, in: IFIP Technical Conference on Optimization Techniques, Springer, 1975, pp. 54-62. 
[33] J. Simon, Differentiation with respect to the domain in boundary value problems, Numerical Functional Analysis and Optimization 2 (7-8) (1980) 649-687.

[34] J. Hadamard, Mémoire sur le problème d'analyse relatif à l'équilibre des plaques élastiques encastrées, Vol. 33, Imprimerie nationale, 1908

[35] M. Delfour, J.-P. Zolésio, Shapes and geometries, volume 22 of advances in design and control, Society for Industrial and Applied Mathematics (SIAM), Philadelphia, PA,

[36] F. de Gournay, Velocity extension for the level-set method and multiple eigenvalues in shape optimization, SIAM Journal on Control and Optimization 45 (1) (2006) 343-367.

[37] J. A. Sethian, A. Wiegmann, Structural boundary design via level set and immersed interface methods, Journal of computational physics 163 (2) (2000) 489-528.

[38] S. O. R. Fedkiw, S. Osher, Level set methods and dynamic implicit surfaces, Surfaces 44 (2002) 77.

[39] J. A. Sethian, Level set methods and fast marching methods: Evolving interfaces in computational geometry, fluid mechanics, computer vision, and materials science, Vol. 3, Cambridge University Press, 1999.

[40] C. D. Johnson, D. A. Kienholz, Finite element prediction of damping in structures with constrained viscoelastic layers, AIAA journal 20 (9) (1982) 1284-1290.

[41] E. Daya, M. Potier-Ferry, A numerical method for nonlinear eigenvalue problems application to vibrations of viscoelastic structures, Computers \& Structures 79 (5) (2001) 533-541.

[42] Y. Koutsawa, I. Charpentier, M. Cherkaoui, et al., A generic approach for the solution of nonlinear residual equations. part i: The diamant toolbox, Computer Methods in Applied Mechanics and Engineering 198 (3-4) (2008) 572-577.

[43] K. Schreiber, Nonlinear eigenvalue problems: Newton-type methods and nonlinear Rayleigh functionals, Ph.D. thesis, Technische Universität Berlin (2008).

[44] M. Hamdaoui, K. Akoussan, E. M. Daya, Comparison of non-linear eigensolvers for modal analysis of frequency dependent laminated visco-elastic sandwich plates, Finite Elements in Analysis and Design 121 (2016) 75 - 85. doi:https://doi. org/10.1016/j.finel.2016.08.001

[45] F. Hecht, New development in Freefem++, Journal of Numerical Mathematics 20 (3-4) (2012) 251-265.

[46] F. Jouve, Xd3d, http://www.cmap.polytechnique.fr/ jouve/xd3d/\#Contact.

[47] M. Dauge, M. Suri, Numerical approximation of the spectra of non-compact operators arising in buckling problems, Journal of Numerical Mathematics 10 (3) (2002) 193-219.

[48] C. Xu, M.-Z. Wu, M. Hamdaoui, Mixed integer multi-objective optimization of composite structures with frequencydependent interleaved viscoelastic damping layers, Computers \& Structures 172 (2016) 81-92. 